\title{
Stability of stealth magnetic field in de Sitter spacetime
}

\author{
Shinji Mukohyama \\ Center for Gravitational Physics, Yukawa Institute for Theoretical Physics, \\ Kyoto University, 606-8502, Kyoto, Japan \\ and Kavli Institute for the Physics and Mathematics of the Universe (WPI), \\ The University of Tokyo Institutes for Advanced Study, \\ The University of Tokyo, Kashiwa, Chiba 277-8583, Japan
}

(Received 4 September 2018; published 28 November 2018)

\begin{abstract}
A detailed stability analysis is presented for the de Sitter solution with a homogeneous magnetic field that was recently found in the context of a $U(1)$ gauge theory nonminimally coupled to scalar-tensor gravity. The magnetic field is "stealth" in the sense that the corresponding stress-energy tensor is of the form of an effective cosmological constant and thus is isotropic despite the fact that the magnetic field has a preferred spatial direction. We study the stability of the solution against linear perturbations in the subhorizon and superhorizon limits. We then present some explicit examples that satisfy all stability conditions. The stable de Sitter solution with a homogeneous magnetic field opens up a new possibility for inflationary magnetogenesis, in which magnetic fields in the Universe at all scales may originate from a classical, homogeneous magnetic field sustained during inflation.
\end{abstract}

DOI: 10.1103/PhysRevD.98.104053

\section{INTRODUCTION}

The origin of magnetic fields in the Universe at various scales is one of the mysteries in modern cosmology. There thus have been a large number of attempts to find a theoretical framework in which magnetic fields in the Universe are generated in the course of the cosmic history (see Refs. [1-4] for reviews). The author recently proposed a new scenario of inflationary magnetogenesis in which magnetic fields in the Universe at all scales may originate from a classical, homogeneous magnetic field sustained during inflation [5]. It was found that a $U(1)$ gauge theory nonminimally coupled to scalar-tensor gravity admits a cosmological attractor solution that represents a de Sitter universe with a homogeneous magnetic field, fully taking into account the backreaction of the magnetic field on the geometry.

In the standard Einstein-Maxwell theory, a homogeneous magnetic field would introduce anisotropies in the geometry through the stress-energy tensor, making it impossible to admit a de Sitter solution. One thus needs to modify the standard Einstein-Maxwell theory one way or another to make a homogeneous magnetic field and an isotropic expansion of the Universe consistent with each other. In

Published by the American Physical Society under the terms of the Creative Commons Attribution 4.0 International license. Further distribution of this work must maintain attribution to the author(s) and the published article's title, journal citation, and DOI. Funded by SCOAP ${ }^{3}$. the solution found Ref. [5], a homogeneous magnetic field has a preferred spatial direction, but its stress-energy tensor is isotropic and is of the form of an effective cosmological constant. In this sense, the magnetic field in the solution is "stealth."

The basic idea behind the model of Ref. [5] is as follows. For simplicity, let us first consider an action for a $U(1)$ gauge field $A_{\mu}$ of the form

$$
I_{w}=\int d^{4} x \sqrt{-g} f(w), \quad w=-\frac{1}{4} F_{\mu \nu} F^{\mu \nu},
$$

where $F_{\mu \nu}=\partial_{\mu} A_{\nu}-\partial_{\nu} A_{\mu}$ is the field strength and $g_{\mu \nu}$ is the spacetime metric. By taking the variation of the action with respect to $g_{\mu \nu}$, one obtains the corresponding stressenergy tensor as

$$
T_{w}^{\mu \nu}=\frac{2}{\sqrt{-g}} \frac{\delta I_{w}}{\delta g_{\mu \nu}}=f^{\prime}(w) F_{\rho}^{\mu} F^{\nu \rho}+f(w) g^{\mu \nu} .
$$

If $f^{\prime}(w)=0$, then the stress-energy tensor is proportional to $g^{\mu \nu}$ and thus admits an exact de Sitter solution. For example, this would be the case if $f(w)=f_{0}+f_{2}\left(w-w_{0}\right)^{2}$ with $f_{0}, f_{2}$, and $w_{0}$ constant and if $w=w_{0}$. However, in Friedmann-Lemaître-Robertson-Walker (FLRW) backgrounds (including the de Sitter spacetime), $w$ for a homogeneous magnetic field decays as $1 / a^{4}$, where $a$ is the scale factor, and thus the only constant value of $w$ that is consistent with the expansion of the Universe is zero. For this reason, this simple action does not work. Let us next consider an action of the form 
$I_{W}=\int d^{4} x \sqrt{-g} f(W), \quad W=-\frac{1}{4} e^{2 \phi} F_{\mu \nu} F^{\mu \nu}$,

where $\phi$ is a scalar field and it is understood that some kinetic terms for $\phi$ are also added to the action. In this case, the corresponding stress-energy tensor is again proportional to $g^{\mu \nu}$ if $f^{\prime}(W)=0$. The main difference from the previous case is that $W$ for a homogeneous magnetic field is proportional to $e^{2 \phi} / a^{4}$ in FLRW backgrounds and thus is constant if $e^{\phi} \propto a^{2}$. This is the basic idea behind the model of Ref. [5].

The model action of Ref. [5], however, includes not only a nonlinear function of $W$ but also other terms such as Horndeski's nonminimal vector coupling and the Horndeski scalar terms. This is because, while the simple action (1.3) supplemented by kinetic terms for $\phi$ in principle admits a de Sitter solution with a homogeneous magnetic field, the stability of such a solution requires inclusion of additional terms in the action.

The purpose of the present paper is to show a detailed stability analysis for the solution representing a de Sitter spacetime with a stealth homogeneous magnetic field. In particular, we study the stability of the solution against linear perturbations in the subhorizon and superhorizon limits. We show that the stability of the solution requires inclusion of Horndeski's nonminimal vector coupling as well as the Horndeski scalar terms. We then present some explicit examples that satisfy all stability conditions.

The rest of the present paper is organized as follows. In Sec. II, we briefly review the model and the solutions studied in Ref. [5], focusing on those without the electric field. In Sec. III, we formulate the linear perturbation analysis and study the stability of the de Sitter solution with the stealth magnetic field against subhorizon perturbations. In Sec. IV, we then study the stability of the solution against superhorizon perturbations. In Sec. V, after showing a couple of classes of models that are unstable, we present explicit models that satisfy all stability conditions. Section VI is then devoted to a summary of the paper and some discussions. Appendixes A-D show explicit expressions of some matrices and coefficients.

\section{REVIEW OF THE MODEL}

In this section, we briefly review the model and the de Sitter solution with a homogeneous magnetic field studied in Ref. [5].

\section{A. Action}

The model consists of a metric $g_{\mu \nu}$, a $U(1)$ gauge field $A_{\mu}$ and a scalar field $\phi$, described by the action

$$
I=\int d^{4} x \sqrt{-g}\left[L+L_{3}+L_{4}+L_{5}+L_{\mathrm{H}}\right],
$$

where $L=L(X, W, Y, Z)$ is an arbitrary function of

$$
\begin{aligned}
X & \equiv-\frac{1}{2} g^{\mu \nu} \partial_{\mu} \phi \partial_{\nu} \phi, \quad W \equiv-\frac{1}{4} \mathcal{F}_{\mu \nu} \mathcal{F}^{\mu \nu}, \\
Y & \equiv \mathcal{F}_{\mu \nu} \tilde{\mathcal{F}}^{\mu \nu}, \quad Z \equiv \mathcal{F}^{\rho \mu} \mathcal{F}_{\rho}^{\nu} \partial_{\mu} \phi \partial_{\nu} \phi ;
\end{aligned}
$$

$\mathcal{F}_{\mu \nu}$ and $\tilde{\mathcal{F}}^{\mu \nu}$ are defined by

$$
\begin{aligned}
\mathcal{F}_{\mu \nu} & \equiv e^{\phi} F_{\mu \nu}, \quad \tilde{\mathcal{F}}^{\mu \nu} \equiv e^{\phi} \tilde{F}^{\mu \nu}, \\
F_{\mu \nu} & \equiv \partial_{\mu} A_{\nu}-\partial_{\nu} A_{\mu}, \quad \tilde{F}^{\mu \nu} \equiv \frac{1}{2} \epsilon^{\mu \nu \rho \sigma} F_{\rho \sigma},
\end{aligned}
$$

and $\epsilon^{0123}=-1 / \sqrt{-g}$;

$L_{3}=-G_{3}(X) \square \phi$,

$L_{4}=G_{4}(X) R+G_{4 X}(X)\left[(\square \phi)^{2}-\left(\nabla^{\mu} \nabla_{\nu} \phi\right)\left(\nabla^{\nu} \nabla_{\mu} \phi\right)\right]$,

$L_{5}=G_{5}(X) G^{\mu \nu} \nabla_{\mu} \nabla_{\nu} \phi-\frac{1}{6} G_{5 X}(X)\left[(\square \phi)^{3}\right.$

$$
\begin{aligned}
& -3(\square \phi)\left(\nabla^{\mu} \nabla_{\nu} \phi\right)\left(\nabla^{\nu} \nabla_{\mu} \phi\right) \\
& \left.+2\left(\nabla^{\mu} \nabla_{\nu} \phi\right)\left(\nabla^{\nu} \nabla_{\rho} \phi\right)\left(\nabla^{\rho} \nabla_{\mu} \phi\right)\right],
\end{aligned}
$$

are shift-symmetric Horndeski scalar terms [6,7]; and

$$
L_{\mathrm{H}}=\xi \tilde{\mathcal{F}}^{\mu \nu} \tilde{\mathcal{F}}^{\rho \sigma} R_{\mu \nu \rho \sigma},
$$

is a simple modification $\left(\tilde{F}^{\mu \nu} \rightarrow \tilde{\mathcal{F}}^{\mu \nu}\right)$ of the Horndeski's nonminimal coupling of the $U(1)$ gauge field to the Riemann tensor $R_{\nu \rho \sigma}^{\mu}$ of the metric $g_{\mu \nu}$ [8]. Here, the scalar field $\phi$ is normalized so that its mass dimension is zero, $G_{3,4,5}(X)$ are arbitrary functions of $X$, the subscript $X$ denotes derivative with respect to $X$, and $\xi$ is an arbitrary constant.

The action is diffeomorphism invariant and enjoys the $U(1)$ gauge symmetry

$$
A_{\mu} \rightarrow A_{\mu}+\partial_{\mu} \lambda
$$

where $\lambda$ is an arbitrary function. Furthermore, the action respects the global symmetry

$$
\phi \rightarrow \phi+\phi_{0}, \quad A_{\mu} \rightarrow e^{-\phi_{0}} A_{\mu},
$$

where $\phi_{0}$ is an arbitrary constant. We assume that the function $L(X, W, Y, Z)$ is even with respect to $Y$,

$$
L(X, W, Y, Z)=L(X, W,-Y, Z) .
$$

This assumption ensures that the equations of motion admit a solution without the electric field [5]. 


\section{B. Bianchi I solution with magnetic field}

We first consider a Bianchi I spacetime

$$
\begin{aligned}
g_{\mu \nu} d x^{\mu} d x^{\nu}= & -N(t)^{2} d t^{2}+a(t)^{2}\left[e^{4 \sigma(t)} d x^{2}\right. \\
& \left.+e^{-2 \sigma(t)}\left(d y^{2}+d z^{2}\right)\right],
\end{aligned}
$$

with

$$
\frac{\dot{a}}{N a}=\text { const } \equiv H_{0}, \quad \frac{\dot{\sigma}}{N}=\text { const } \equiv \Sigma_{0},
$$

where an overdot represents a derivative with respect to $t$. We further assume that the scalar field is homogeneous, $\phi=\phi(t)$, and has a constant "velocity" as

$$
\frac{\dot{\phi}}{N}=\text { const }
$$

and that the $U(1)$ gauge field represents a homogeneous magnetic field as

$$
A_{t}=A_{x}=0, \quad A_{y}=\frac{1}{2} B z, \quad A_{z}=-\frac{1}{2} B y,
$$

where $B$ is a constant.

Assuming that not only $H_{0}, \Sigma_{0}, B$, and $\dot{\phi} / N$ but also $\chi \equiv e^{\phi} / a^{2}$ are constant [see the sentences just after (1.3) for the motivation for this extra assumption] and rescaling the spatial coordinates so that $\chi=1$, the equations of motion are reduced to

$$
0=b L_{Y},
$$

and

$$
\begin{aligned}
0= & 16(1-s)^{6} H_{0}^{6} G_{5 X}-6 G_{4}(1-s)^{2} H_{0}^{2}-L \\
& +4\left[4 \xi b^{2}+6(1-s)^{2} G_{4 X}\right](1-s)^{2} H_{0}^{4}, \\
0= & 8 G_{5 X X}(1+2 s)(1-s)^{6} H_{0}^{6} \\
& +6(1-s)^{4}\left[4(1+s) G_{4 X X}+(1+4 s) G_{5 X}\right] H_{0}^{4} \\
& +2\left\{-(1-4 s) \xi b^{2}+3(1-s)^{2}\left[G_{3 X}\right.\right. \\
& \left.\left.+(1+3 s) G_{4 X}\right]\right\} H_{0}^{2}-3 s G_{4}+(1-s) L_{X}, \\
0= & 4\left[18 s(1-s)^{2} G_{4 X}-\xi\left(5-4 s+8 s^{2}\right) b^{2}\right] H_{0}^{2} \\
& +72 G_{5 X}(1-s)^{4} s H_{0}^{4}-b^{2} L_{W}-18 s G_{4},
\end{aligned}
$$

where $b \equiv B / H_{0}$ and $s \equiv \Sigma_{0} / H_{0}$. Hereafter, subscripts $W$, $Y$, and $Z$ represent derivatives with respect to them. Since $Y=0$ for the ansatz (2.12) and we have assumed (2.8), the first equation (2.13) is automatically satisfied. Therefore, we have three algebraic equations (2.14) to be solved with respect to the three parameters $\left(H_{0}, b, s\right)$ of the ansatz. Generically, they admit a solution (or a set of solutions).

\section{De Sitter solution with magnetic field}

By fine tuning one of parameters in the action, one can then take the limit $s \rightarrow 0$ so that the spacetime becomes a de Sitter,

$g_{\mu \nu} d x^{\mu} d x^{\nu}=-N(t)^{2} d t^{2}+a(t)^{2}\left(d x^{2}+d y^{2}+d z^{2}\right)$.

Then, under the assumption (2.8), the independent equations of motion are

$0=16 G_{5 X} H_{0}^{6}+8\left(2 \xi b^{2}+3 G_{4 X}\right) H_{0}^{4}-6 G_{4} H_{0}^{2}-L$,

$0=8 G_{5 X X} H_{0}^{6}+6\left(4 G_{4 X X}+G_{5 X}\right) H_{0}^{4}$

$$
+2\left[-\xi b^{2}+3\left(G_{3 X}+G_{4 X}\right)\right] H_{0}^{2}+L_{X},
$$

$0=20 \xi H_{0}^{2}+L_{W}$.

These are three algebraic equations. One of the three equations (or one combination of them) simply represents the fine-tuning of one of parameters in the action, and the remaining two equations can generically be solved with respect to the two parameters $\left(H_{0}, b\right)$ of the ansatz.

If we relax the fine-tuning without abandoning the discrete symmetry (2.8), then the solution goes back to the Bianchi I solution with the three parameters $\left(H_{0}, b, s\right)$ described in the previous subsection.

\section{Attractor condition}

Reference [5] found the necessary and sufficient condition under which the de Sitter solution with a homogeneous magnetic field introduced in Sec. II C is stable against homogeneous linear perturbations, under the assumption (2.8). The stability condition, or the attractor condition, is $\mathcal{A} / \mathcal{N}>0$, where

$$
\begin{aligned}
\mathcal{N}= & 2 \zeta_{3} g_{h}\left(\zeta_{3}-8 \zeta_{1}\right) b^{2}+\zeta_{1}\left(\zeta_{1} \zeta_{2}+3 \zeta_{3}^{2}\right), \\
\mathcal{A}= & 56 b^{6} g_{h}^{3}-4\left(9 \zeta_{1}+\zeta_{2}+15 \zeta_{3}\right) g_{h}^{2} b^{4}-2 g_{h} \zeta_{4}\left(\zeta_{1}-\zeta_{3}\right) b^{3} \\
& +\left[6\left(-\zeta_{1}^{2}+\zeta_{1} \zeta_{2}+2 \zeta_{1} \zeta_{3}+2 \zeta_{3}^{2}\right) g_{h}\right. \\
& \left.+\zeta_{5}\left(\zeta_{1}-\zeta_{3}\right)^{2}\right] b^{2}+\frac{3}{2} \zeta_{1} \zeta_{4}\left(\zeta_{1}-\zeta_{3}\right) b
\end{aligned}
$$

$g_{h}$ and $\zeta_{i}(i=1, \cdots 5)$ are constants defined by

$$
\begin{aligned}
g_{h}= & \xi H_{0}^{2} / M_{\mathrm{Pl}}^{2}, \quad \zeta_{1}=2 b^{2} g_{h}+g_{4}-4 g_{4 x}-4 g_{5 x} \\
\zeta_{2}= & 2 b^{2} g_{h}+6 g_{3 x}+24 g_{3 x x}+72 g_{4 x x}+96 g_{4 x x x} \\
& +6 g_{5 x}+48 g_{5 x x}+32 g_{5 x x x}+4 l_{x x} \\
\zeta_{3}= & 4 b^{2} g_{h}+2 g_{3 x}+4 g_{4 x}+16 g_{4 x x}+6 g_{5 x}+8 g_{5 x x} \\
\zeta_{4}= & -4\left(g_{h}+l_{x w}\right) b, \quad \zeta_{5}=-12 g_{h}-b^{2} l_{w w} ;
\end{aligned}
$$

and 


$$
\begin{aligned}
L_{X X} & =l_{x x} \frac{M_{\mathrm{Pl}}^{2}}{H_{0}^{2}}, \quad L_{X W}=l_{x w} \frac{M_{\mathrm{Pl}}^{2}}{H_{0}^{2}}, \quad L_{W W}=l_{w w} \frac{M_{\mathrm{Pl}}^{2}}{H_{0}^{2}}, \\
G_{3 X} & =g_{3 x} \frac{M_{\mathrm{Pl}}^{2}}{H_{0}^{2}}, \quad G_{3 X X}=g_{3 x x} \frac{M_{\mathrm{Pl}}^{2}}{H_{0}^{4}}, \\
G_{4} & =g_{4} M_{\mathrm{Pl}}^{2}, \quad G_{4 X}=g_{4 x} \frac{M_{\mathrm{Pl}}^{2}}{H_{0}^{2}}, \quad G_{4 X X}=g_{4 x x} \frac{M_{\mathrm{Pl}}^{2}}{H_{0}^{4}}, \\
G_{4 X X X} & =g_{4 x x x} \frac{M_{\mathrm{Pl}}^{2}}{H_{0}^{6}}, \quad G_{5 X}=g_{5 x} \frac{M_{\mathrm{Pl}}^{2}}{H_{0}^{4}}, \\
G_{5 X X} & =g_{5 x x} \frac{M_{\mathrm{Pl}}^{2}}{H_{0}^{6}}, \quad G_{5 X X X}=g_{5 x x x} \frac{M_{\mathrm{Pl}}^{2}}{H_{0}^{8}}
\end{aligned}
$$

It is understood that the left-hand sides of (2.19) are evaluated at the de Sitter solution with a homogeneous magnetic field and thus are constant.

We shall later see that the absence of ghosts implies that $\mathcal{N}>0$. Therefore, the attractor condition is

$$
\mathcal{A}>0 \text {. }
$$

\section{LINEAR PERTURBATION}

In this section, we consider inhomogeneous linear perturbations around the de Sitter attractor solution with a homogeneous magnetic field $\left(H_{0}>0, s=0, b \neq 0\right)$ and seek the no-ghost conditions and the sound speeds in the subhorizon limit.

\section{A. Decomposition of linear perturbation}

Around the general background introduced in Sec. II B, we introduce the components $\Phi, V_{i}$ and $h_{i j}(i, j=1,2,3)$ of the metric perturbation $\delta g_{\mu \nu}$, the components $\delta A_{a}$ $(a=0, \ldots, 3)$ of the perturbation of the vector field $\delta A_{\mu}$, and the perturbation $\pi$ of the scalar field $\phi$ as

$$
\begin{aligned}
\delta g_{\mu \nu} d x^{\mu} d x^{\nu} & =-2 \Phi e^{0} e^{0}+V_{i} e^{0} e^{i}+V_{i} e^{i} e^{0}+h_{i j} e^{i} e^{j}, \\
\delta A_{\mu} d x^{\mu} & =\delta A_{a} e^{a}, \quad \delta \phi=\pi,
\end{aligned}
$$

where

$$
\begin{aligned}
& e^{0}=N(t) d t, \quad e^{1}=a(t) e^{2 \sigma(t)} d x, \\
& e^{2}=a(t) e^{-\sigma(t)} d y, \quad e^{3}=a(t) e^{-\sigma(t)} d z,
\end{aligned}
$$

and the Einstein's summation rule is employed. We then decompose each component of the perturbation into spatial Fourier modes. For this purpose, we first introduce scalar harmonics $Y_{n}(n=c c, c s, s c, s s)$ as

$$
\begin{aligned}
& Y_{c c}=\cos \left(k_{x} x\right) \cos \left(k_{y} y+k_{z} z\right), \\
& Y_{c s}=\cos \left(k_{x} x\right) \sin \left(k_{y} y+k_{z} z,\right. \\
& Y_{s c}=\sin \left(k_{x} x\right) \cos \left(k_{y} y+k_{z} z\right), \\
& Y_{s s}=\sin \left(k_{x} x\right) \sin \left(k_{y} y+k_{z} z\right),
\end{aligned}
$$

and then define odd and even vector harmonics $Y_{n, p}^{\text {odd/even }}$ $(n=c c, c s, s c, s s ; p, q=2,3)$ as

$$
Y_{n, p}^{\text {odd }}=\epsilon_{p q} \frac{\partial}{\partial x^{q}} Y_{n}, \quad Y_{n, p}^{\text {even }}=\frac{\partial}{\partial x^{p}} Y_{n}
$$

where $x^{2}=y, x^{3}=z$, and $\epsilon_{p q}$ is the two-dimensional LeviCività symbol $\left(\epsilon_{23}=-\epsilon_{32}=1\right.$ and $\left.\epsilon_{22}=\epsilon_{33}=0\right)$, and odd and even tensor harmonics $Y_{n, p q}^{\text {odd/even }}(n=c c, c s, s c$, ss; $p, q=2,3)$ as

$$
Y_{n, p q}^{\mathrm{odd} / \mathrm{even}}=\frac{1}{2}\left(\frac{\partial}{\partial x^{p}} Y_{n, q}^{\mathrm{odd} / \mathrm{even}}+\frac{\partial}{\partial x^{q}} Y_{n, p}^{\mathrm{odd} / \mathrm{even}}\right) .
$$

The components of the perturbations are then decomposed as

$$
\begin{aligned}
\pi & =\int \frac{d^{3} k}{(2 \pi)^{3}} \pi_{n} Y_{n}, \quad \Phi=\int \frac{d^{3} k}{(2 \pi)^{3}} \Phi_{n} Y_{n}, \\
V_{1} & =\int \frac{d^{3} k}{(2 \pi)^{3}} \chi_{n} Y_{n}, \quad h_{11}=\int \frac{d^{3} k}{(2 \pi)^{3}} \psi_{n} Y_{n}, \\
\delta A_{0} & =\int \frac{d^{3} k}{(2 \pi)^{3}} A_{0, n} Y_{n}, \quad \delta A_{1}=\int \frac{d^{3} k}{(2 \pi)^{3}} A_{1, n} Y_{n}, \\
V_{p} & =\int \frac{d^{3} k}{(2 \pi)^{3}}\left(V_{n}^{\text {odd }} Y_{n, p}^{\text {odd }}+V_{n}^{\text {even }} Y_{n, p}^{\text {even }}\right), \\
h_{1 p} & =h_{p 1}=\int \frac{d^{3} k}{(2 \pi)^{3}}\left(h_{1, n}^{\text {odd }} Y_{n, p}^{\text {odd }}+\beta_{n} Y_{n, p}^{\text {even }}\right), \\
\delta A_{p} & =\int \frac{d^{3} k}{(2 \pi)^{3}}\left(A_{n}^{\text {odd }} Y_{n, p}^{\text {odd }}+A_{n}^{\text {even }} Y_{n, p}^{\text {even }}\right), \\
h_{p q} & =\int \frac{d^{3} k}{(2 \pi)^{3}}\left(h_{n}^{\text {odd }} Y_{n, p q}^{\text {odd }}+E_{n} Y_{n, p q}^{\text {even }}+\tau_{n} Y_{n} \delta_{p q}\right),
\end{aligned}
$$

where $p, q=2,3$ and the Einstein's summation rule for $n=c c, c s, s c, s s$ is understood.

We fix gauge freedom associated with the spacetime coordinate transformation and the $U(1)$ gauge transformation as

$$
\beta_{n}=h_{n}^{\text {odd }}=E_{n}=\tau_{n}=0, \quad A_{n}^{\text {even }}=0 .
$$

\section{B. General quadratic action}

Substituting the decomposition of perturbations to the action, imposing the gauge condition, and expanding the action up to the quadratic order in perturbations, we obtain the quadratic action for the linear perturbations around a general background introduced in Sec. II B as 


$$
\begin{aligned}
I^{(2)}= & \frac{1}{2} \int \frac{d^{3} k}{(2 \pi)^{3}} \int N a^{3} d t\left[\frac{1}{N^{2}} \dot{\mathbf{Y}}_{n}^{\top} \mathbf{K} \dot{\mathbf{Y}}_{n}+\frac{1}{N}\left(\dot{\mathbf{Y}}_{n}^{\top} \mathbf{M} \mathbf{Y}_{n}\right.\right. \\
& \left.+\mathbf{Y}_{n}^{\top} \mathbf{M}^{\top} \dot{\mathbf{Y}}_{n}\right)-\mathbf{Y}_{n}^{\top} \mathbf{V} \mathbf{Y}_{n}+\left(\mathbf{Z}_{n}^{\top} \mathbf{A} \mathbf{Y}_{n}+\mathbf{Y}_{n}^{\top} \mathbf{A}^{\top} \mathbf{Z}_{n}\right) \\
& \left.+\frac{1}{N}\left(\mathbf{Z}_{n}^{\top} \mathbf{B} \dot{\mathbf{Y}}_{n}+\dot{\mathbf{Y}}_{n}^{\top} \mathbf{B}^{\top} \mathbf{Z}_{n}\right)+\mathbf{Z}_{n}^{\top} \mathbf{C} \mathbf{Z}_{n}\right],
\end{aligned}
$$

where the superscript $T$ represents the transpose operation,

$$
\mathbf{Y}_{n}=\left(\begin{array}{c}
k_{\perp} a^{2} A_{n}^{\text {odd }} \\
k_{\perp} h_{1, n}^{\text {odd }} \\
a^{2} A_{1, n} \\
\pi_{n} \\
\psi_{n}
\end{array}\right)
$$

represents dynamical degrees of freedom,

$$
\begin{aligned}
\mathbf{Z}_{c c}=\left(\begin{array}{c}
k_{\perp} V_{s c}^{\text {odd }} \\
k_{\perp} V_{c c}^{\text {even }} \\
a^{2} A_{0, s c} \\
\chi_{s c} \\
\frac{a}{k_{\perp}} H_{0} \Phi_{c c}
\end{array}\right), & \mathbf{Z}_{c s}=\left(\begin{array}{c}
k_{\perp} V_{s s}^{\text {odd }} \\
k_{\perp} V_{c s}^{\text {even }} \\
a^{2} A_{0, s s} \\
\chi_{s s} \\
\frac{a}{k_{\perp}} H_{0} \Phi_{c s}
\end{array}\right), \\
\mathbf{Z}_{s c}=\left(\begin{array}{c}
-k_{\perp} V_{c c}^{\text {odd }} \\
k_{\perp} V_{s c}^{\text {even }} \\
-a^{2} A_{0, c c} V_{c s}^{\text {odd }} \\
-\chi_{c c} \\
\frac{a}{k_{\perp}} H_{0} \Phi_{s c}
\end{array}\right), & \mathbf{Z}_{s s}=\left(\begin{array}{c}
k_{\perp} V_{s s}^{\text {even }} \\
-a^{2} A_{0, c s} \\
-\chi_{c s} \\
\frac{a}{k_{\perp}} H_{0} \Phi_{s s}
\end{array}\right)
\end{aligned}
$$

represent nondynamical degrees of freedom, $k_{\perp}=\sqrt{\left(k_{y}\right)^{2}+\left(k_{z}\right)^{2}}$, and the Einstein's summation rule for $n=c c, c s, s c, s s$ is again understood. Important points are that the matrices $\mathbf{K}, \mathbf{M}, \mathbf{V}, \mathbf{A}, \mathbf{B}$, and $\mathbf{C}$ are common for all $n=c c, c s, s c, s s$ and that there are no couplings between $\left(Y_{n}, Z_{n}\right)$ and $\left(Y_{n^{\prime}}, Z_{n^{\prime}}\right)$ with $n \neq n^{\prime}$. In general, however, even and odd sectors within $\left(Y_{n}\right.$, $Z_{n}$ ) couple with each other.

Integrating out the nondynamical variables $\mathbf{Z}_{n}$, one obtains the quadratic action for the dynamical variables $\mathbf{Y}_{n}$ as

$$
\begin{aligned}
\tilde{I}^{(2)}= & \frac{1}{2} \int \frac{d^{3} k}{(2 \pi)^{3}} \int N a^{3} d t\left[\frac{1}{N^{2}} \dot{\mathbf{Y}}_{n}^{\top} \overline{\mathbf{K}} \dot{\mathbf{Y}}_{n}+\frac{1}{N}\left(\dot{\mathbf{Y}}_{n}^{\top} \overline{\mathbf{M}} \mathbf{Y}_{n}\right.\right. \\
& \left.\left.+\mathbf{Y}_{n}^{\top} \overline{\mathbf{M}} \dot{\mathbf{Y}}_{n}\right)-\mathbf{Y}_{n}^{\top} \overline{\mathbf{V}} \mathbf{Y}_{n}\right],
\end{aligned}
$$

where

$$
\begin{aligned}
& \overline{\mathbf{K}}=\mathbf{K}-\mathbf{B}^{\top} \mathbf{C}^{-1} \mathbf{B}, \quad \overline{\mathbf{M}}=\frac{1}{2}\left(\overline{\mathbf{m}}-\overline{\mathbf{m}}^{\top}\right), \\
& \overline{\mathbf{V}}=\mathbf{V}+\mathbf{A}^{\top} \mathbf{C}^{-1} \mathbf{A}+\frac{1}{2 N a^{3}} \partial_{t}\left[a^{3}\left(\overline{\mathbf{m}}+\overline{\mathbf{m}}^{\top}\right)\right],
\end{aligned}
$$

and

$$
\overline{\mathbf{m}}=\mathbf{M}-\mathbf{B}^{\mathrm{\top}} \mathbf{C}^{-1} \mathbf{A},
$$

It is then straightforward to diagonalize the kinetic matrix $\overline{\mathbf{K}}$ as

$$
\overline{\mathbf{K}} \rightarrow \tilde{\mathbf{K}}=\mathbf{U}^{\top} \overline{\mathbf{K}} \mathbf{U}, \quad \mathbf{U}=\mathbf{U}_{1} \mathbf{U}_{2} \mathbf{U}_{3} \mathbf{U}_{4} \mathbf{U}_{5},
$$

where

$$
\begin{aligned}
& \mathbf{U}_{1}=\left(\begin{array}{ccccc}
1 & -\frac{\bar{K}_{12}}{\bar{K}_{11}} & -\frac{\bar{K}_{13}}{\bar{K}_{11}} & -\frac{\bar{K}_{14}}{\bar{K}_{11}} & -\frac{\bar{K}_{15}}{\bar{K}_{11}} \\
0 & 1 & 0 & 0 & 0 \\
0 & 0 & 1 & 0 & 0 \\
0 & 0 & 0 & 1 & 0 \\
0 & 0 & 0 & 0 & 1
\end{array}\right), \\
& \mathbf{U}_{2}=\left(\begin{array}{ccccc}
1 & 0 & 0 & 0 & 0 \\
0 & 1 & -\frac{\bar{K}_{23}}{\bar{K}_{22}} & -\frac{\bar{K}_{24}}{\bar{K}_{22}} & -\frac{\bar{K}_{25}}{\bar{K}_{22}} \\
0 & 0 & 1 & 0 & 0 \\
0 & 0 & 0 & 1 & 0 \\
0 & 0 & 0 & 0 & 1
\end{array}\right) \text {, } \\
& \mathbf{U}_{3}=\left(\begin{array}{ccccc}
1 & 0 & 0 & 0 & 0 \\
0 & 1 & 0 & 0 & 0 \\
0 & 0 & 1 & -\bar{K}_{34} & -\bar{K}_{33} \\
0 & 0 & 0 & 1 & 0 \\
0 & 0 & 0 & 0 & 1
\end{array}\right), \\
& \mathbf{U}_{4}=\left(\begin{array}{ccccc}
1 & 0 & 0 & 0 & 0 \\
0 & 1 & 0 & 0 & 0 \\
0 & 0 & 1 & 0 & 0 \\
0 & 0 & 0 & 1 & -\frac{\bar{K}_{45}}{\bar{K}_{44}} \\
0 & 0 & 0 & 0 & 1
\end{array}\right),
\end{aligned}
$$

and $\quad \mathbf{U}_{5}=\operatorname{diag}\left(1, k / k_{\perp}, k / k_{\perp}, 1, k^{2} / k_{\perp}^{2}\right)$. Here, $\quad k=$ $\sqrt{\left(k_{x}\right)^{2}+\left(k_{\perp}\right)^{2}}$. Correspondingly, the friction matrix $\overline{\mathbf{M}}$ and the mass matrix $\overline{\mathbf{V}}$ transform as

$$
\begin{aligned}
\overline{\mathbf{M}} \rightarrow \tilde{\mathbf{M}}= & \frac{1}{2}\left(\tilde{\mathbf{m}}-\tilde{\mathbf{m}}^{\top}\right), \\
\overline{\mathbf{V}} \rightarrow \tilde{\mathbf{V}}= & \mathbf{U}^{\top} \overline{\mathbf{V}} \mathbf{U}-\frac{1}{N}\left(\dot{\mathbf{U}}^{\top} \overline{\mathbf{M}} \mathbf{U}+\mathbf{U}^{\top} \overline{\mathbf{M}}^{\top} \dot{\mathbf{U}}\right) \\
& -\frac{1}{N^{2}} \dot{\mathbf{U}}^{\top} \overline{\mathbf{K}} \dot{\mathbf{U}}+\frac{1}{2 N a^{3}} \partial_{t}\left[a^{3}\left(\tilde{\mathbf{m}}+\tilde{\mathbf{m}}^{\top}\right)\right],
\end{aligned}
$$

where 


$$
\tilde{\mathbf{m}}=\mathbf{U}^{\top} \overline{\mathbf{M}} \mathbf{U}+\frac{1}{N} \mathbf{U}^{\top} \overline{\mathbf{K}} \dot{\mathbf{U}}
$$

The quadratic action is now written as

$$
\begin{aligned}
& \tilde{I}^{(2)}=\frac{1}{2} \int \frac{d^{3} k}{(2 \pi)^{3}} \int N a^{3} d t\left[\frac{1}{N^{2}} \dot{\tilde{\mathbf{Y}}}_{\mathrm{n}}^{\top} \tilde{\mathbf{K}}_{\tilde{\mathbf{Y}}}\right. \\
& +\frac{1}{N}\left(\dot{\tilde{\mathbf{Y}}}_{\mathrm{n}}^{\top} \tilde{\mathbf{M}} \tilde{\mathbf{Y}}_{\mathrm{n}}+\tilde{\mathbf{Y}}_{\mathrm{n}}^{\top} \tilde{\mathbf{M}}^{\top} \dot{\tilde{\mathbf{Y}}}_{\mathrm{n}}\right)-\tilde{\mathbf{Y}}_{\mathrm{n}}^{\top} \tilde{\mathbf{V}}_{\mathrm{n}} \tilde{\mathbf{Y}}_{\mathrm{n}},
\end{aligned}
$$

where the new variables $\tilde{\mathbf{Y}}_{n}$ are related to the original variables $\mathbf{Y}_{n}$ via $\mathbf{Y}_{n}=\mathbf{U} \tilde{\mathbf{Y}}_{n}$.

\section{Quadratic action around de Sitter background with magnetic field}

In the de Sitter background with a homogeneous magnetic field, the matrices in (3.8) are greatly simplified, and all their components are written in terms of $p_{\perp} \equiv k_{\perp} / a, p_{x} \equiv k_{x} / a$, $b, g_{h}, g_{4}$, and $\zeta_{\alpha}(\alpha=1, \ldots, 7)$, where $g_{h}$ and $\zeta_{\alpha}$ are defined in (2.18) and

$$
\begin{aligned}
& \zeta_{6}=4\left(-3 g_{h}+2 l_{z}\right), \\
& \zeta_{7}=4\left(-3 g_{h}+2 l_{z}+4 b^{2} l_{y y}\right) .
\end{aligned}
$$

Here, $l_{z}$ and $l_{y y}$ are defined as

$$
L_{Z}=l_{z} \frac{M_{\mathrm{Pl}}^{2}}{H_{0}^{2}}, \quad L_{Y Y}=l_{y y} \frac{M_{\mathrm{Pl}}^{2}}{H_{0}^{2}},
$$

where it is understood that the left-hand sides of (3.20) are evaluated at the de Sitter solution with a homogeneous magnetic field. It is convenient to decompose the matrices into submatrices as

$$
\begin{aligned}
\mathbf{K}=\left(\begin{array}{cc}
\mathbf{K}_{\text {odd }} & \mathbf{0} \\
\mathbf{0} & \mathbf{K}_{\text {even }}
\end{array}\right), & \mathbf{M}=\left(\begin{array}{cc}
\mathbf{0} & \mathbf{M}_{\text {mix }} \\
-\mathbf{M}_{\text {mix }}^{\top} & \mathbf{M}_{\text {even }}
\end{array}\right), \\
\mathbf{V}=\left(\begin{array}{cc}
\mathbf{V}_{\text {odd }} & \mathbf{V}_{\text {mix }} \\
\mathbf{V}_{\text {mix }}^{\top} & \mathbf{V}_{\text {even }}
\end{array}\right), & \mathbf{A}=\left(\begin{array}{cc}
\mathbf{A}_{\text {odd }} & \mathbf{A}_{\text {mixI }} \\
\mathbf{A}_{\text {mix II }} & \mathbf{A}_{\text {even }}
\end{array}\right), \\
\mathbf{B}=\left(\begin{array}{cc}
\mathbf{B}_{\text {odd }} & \mathbf{0} \\
\mathbf{B}_{\text {mix }} & \mathbf{B}_{\text {even }}
\end{array}\right), & \mathbf{C}=\left(\begin{array}{cc}
\mathbf{C}_{\text {odd }} & \mathbf{C}_{\text {mix }} \\
\mathbf{C}_{\text {mix }}^{\top} & \mathbf{C}_{\text {even }}
\end{array}\right),
\end{aligned}
$$

where explicit expressions for the submatrices are given in Appendix A.

If $b=0$ (we have already set $s=0$ ), then, since $L$ is assumed to be even with respect to $Y$ in (2.8), the theory and the background respect the parity invariance, and as a result, the mixing matrices $\mathbf{M}_{\text {mix }}$, $\mathbf{V}_{\text {mix }}, \mathbf{A}_{\text {mix I }}, \mathbf{A}_{\text {mix II }}, \mathbf{B}_{\text {mix }}$, and $\mathbf{C}_{\text {mix }}$ vanish. In general, they do not vanish, and thus the even and odd perturbations do mix.

The quadratic action for the dynamical variables $\mathbf{Y}_{n}$ is then given by the formulas (3.11)-(3.13). After diagonalization of the kinetic matrix, one obtains (3.18) with (3.14)-(3.17).

\section{Subhorizon limit}

For the theoretical consistency, one needs to analyze the stability of the background in the UV, i.e., in the subhorizon limit $p^{2} \gg H_{0}^{2}$, where $p=\sqrt{p_{x}^{2}+p_{\perp}^{2}}=k / a$. This ensures the absence of instability of which the timescale is parametrically shorter than the cosmological timescale $\sim 1 / H_{0}$. The stability of the solution against perturbations with $p^{2} \ll H_{0}^{2}$ shall be studied in the next section.

In the subhorizon limit $p^{2} \gg H_{0}^{2}$, introducing a small bookkeeping parameter $\epsilon$ so that $H_{0}^{2} / p^{2}=\mathcal{O}\left(\epsilon^{2}\right)$, components of the matrices in the quadratic action (3.18) are simplified as

$$
\begin{aligned}
\tilde{\mathbf{K}} & =\left(\begin{array}{ccccc}
\tilde{K}_{1} & 0 & 0 & 0 & 0 \\
0 & \tilde{K}_{2} & 0 & 0 & 0 \\
0 & 0 & \tilde{K}_{3} & 0 & 0 \\
0 & 0 & 0 & \tilde{K}_{4} & 0 \\
0 & 0 & 0 & 0 & \tilde{K}_{5}
\end{array}\right)+\mathcal{O}\left(\epsilon^{2}\right), \\
\tilde{\mathbf{M}} & =\left(\begin{array}{ccccc}
0 & 0 & 0 & \tilde{M}_{1} & \tilde{M}_{2} \\
0 & 0 & \tilde{M}_{3} & 0 & 0 \\
0 & -\tilde{M}_{3} & 0 & 0 & 0 \\
-\tilde{M}_{1} & 0 & 0 & 0 & 0 \\
-\tilde{M}_{2} & 0 & 0 & 0 & 0
\end{array}\right)+p \mathcal{O}\left(\epsilon^{2}\right), \\
\tilde{\mathbf{V}} & =\left(\begin{array}{ccccc}
\tilde{V}_{1} & 0 & 0 & 0 & 0 \\
0 & \tilde{V}_{2} & 0 & 0 & 0 \\
0 & 0 & \tilde{V}_{3} & 0 & 0 \\
0 & 0 & 0 & \tilde{V}_{4} & \tilde{V}_{6} \\
0 & 0 & 0 & \tilde{V}_{6} & \tilde{V}_{5}
\end{array}\right)+p^{2} \mathcal{O}\left(\epsilon^{2}\right),
\end{aligned}
$$

where 
$\tilde{K}_{1}=\zeta_{6}$,

$\tilde{K}_{2}=\left(\frac{p_{\perp}^{2}}{\zeta_{1}}+\frac{p_{x}^{2}}{\zeta_{1}-2 b^{2} g_{h}}\right)^{-1} p^{2}$,

$\tilde{K}_{3}=\left(\frac{p_{\perp}^{2}}{\zeta_{7}}+\frac{p_{x}^{2}}{\zeta_{6}}\right)^{-1} p^{2}$,

$\tilde{K}_{4}=\frac{c_{1} p_{\perp}^{4}+2 c_{2} p_{\perp}^{2} p_{x}^{2}+\mathcal{N} p_{x}^{4}}{\left[\left(\zeta_{1}-\zeta_{3}\right) p^{2}+3 b^{2} g_{h} p_{\perp}^{2}\right]^{2}}$,

$\tilde{K}_{5}=\frac{\left(\zeta_{1}-2 b^{2} g_{h}\right) \mathcal{N} p^{4}}{4\left(c_{1} p_{\perp}^{4}+2 c_{2} p_{\perp}^{2} p_{x}^{2}+\mathcal{N} p_{x}^{4}\right)}$,

$\tilde{M}_{1}=\frac{p_{\perp}\left(c_{3} p_{\perp}^{2}+c_{4} p_{x}^{2}\right)}{\left(\zeta_{1}-\zeta_{3}\right) p^{2}+3 b^{2} g_{h} p_{\perp}^{2}}$

$\tilde{M}_{2}=\frac{b g_{h} p_{\perp} p^{2}\left(\zeta_{1}-2 b^{2} g_{h}\right)\left(c_{5} p_{\perp}^{2}+c_{6} p_{x}^{2}\right)}{\zeta_{1}\left(c_{1} p_{\perp}^{4}+2 c_{2} p_{\perp}^{2} p_{x}^{2}+\mathcal{N} p_{x}^{4}\right)}$,

$\tilde{M}_{3}=-\frac{2 b g_{h} p_{\perp}}{\zeta_{1}} \tilde{K}_{2}$,

$\tilde{V}_{1}=\zeta_{5} p_{\perp}^{2}+4 g_{h}\left(\frac{4 b^{2} g_{h}}{\zeta_{1}}-3\right) p_{x}^{2}$,

$\tilde{V}_{2}=g_{4} p^{2}$,

$\tilde{V}_{3}=\frac{4 g_{h} p^{2}\left[3\left(2 b^{2} g_{h}-\zeta_{1}\right) p_{\perp}^{2}+\left(4 b^{2} g_{h}-3 \zeta_{1}\right) p_{x}^{2}\right]}{\left(\zeta_{1}-2 b^{2} g_{h}\right) p_{\perp}^{2}+\zeta_{1} p_{x}^{2}}$,

$\tilde{V}_{4}=\frac{c_{7} p_{\perp}^{6}+c_{8} p_{\perp}^{4} p_{x}^{2}+c_{9} p_{\perp}^{2} p_{x}^{4}+c_{10} p_{x}^{6}}{\left[\left(\zeta_{1}-\zeta_{3}\right) p^{2}+3 b^{2} g_{h} p_{\perp}^{2}\right]^{2}}$,

$\tilde{V}_{5}=\frac{p^{4}\left(c_{11} p_{\perp}^{6}+c_{12} p_{\perp}^{4} p_{x}^{2}+c_{13} p_{\perp}^{2} p_{x}^{4}+c_{14} p_{x}^{6}\right)}{\left[c_{1} p_{\perp}^{4}+2 c_{2} p_{\perp}^{2} p_{x}^{2}+\mathcal{N} p_{x}^{4}\right]^{2}}$,

$\tilde{V}_{6}=\frac{b^{2} g_{h} p_{\perp}^{2} p^{2}\left(c_{15} p_{\perp}^{4}+2 c_{16} p_{\perp}^{2} p_{x}^{2}+c_{17} p_{x}^{4}\right)}{\left[\left(\zeta_{1}-\zeta_{3}\right) p^{2}+3 b^{2} g_{h} p_{\perp}^{2}\right]\left[c_{1} p_{\perp}^{4}+2 c_{2} p_{\perp}^{2} p_{x}^{2}+\mathcal{N} p_{x}^{4}\right]}$.

Here, $\mathcal{N}$ is defined in (2.17), and $c_{1}, \ldots, c_{17}$ are shown in Appendix B.

\section{E. No-ghost conditions}

All components $\tilde{K}_{1,2,3,4,5}$ of the diagonalized kinetic matrix in the subhorizon limit are positive for ${ }^{\forall} p_{\perp}$ and ${ }^{\forall} p_{x}$ such that $p^{2} \neq 0$, if and only if $\mathcal{N}_{a}>0(a=1, \ldots, 7)$, where

$$
\begin{array}{ll}
\mathcal{N}_{1}=\zeta_{1}, & \mathcal{N}_{2}=\zeta_{1}-2 b^{2} g_{h}, \\
\mathcal{N}_{3}=\zeta_{6}, & \mathcal{N}_{4}=\zeta_{7}, \quad \mathcal{N}_{5}=\mathcal{N},
\end{array}
$$

and

$$
\mathcal{N}_{6}=c_{1}, \quad \mathcal{N}_{7}=c_{2}+\sqrt{c_{1} \mathcal{N}}
$$

Here, the positivity of the last three $\mathcal{N}_{5,6,7}$ is necessary and sufficient for $c_{1} p_{\perp}^{4}+2 c_{2} p_{\perp}^{2} p_{x}^{2}+\mathcal{N} p_{x}^{4}$ to be positive for ${ }^{\forall} p_{\perp}$ and ${ }^{\forall} p_{x}$ such that $p^{2} \neq 0$.

One can actually show that

$$
\begin{aligned}
& \mathcal{N}_{6}=\frac{\mathcal{N}_{2}}{4 \mathcal{N}_{1}^{2}}\left(\mathcal{N}_{1}-\mathcal{N}_{2}\right)^{2}\left(4 \mathcal{N}_{1}-\zeta_{3}\right)^{2}+\frac{\mathcal{N}_{5}}{4 \mathcal{N}_{1}^{2}}\left(\mathcal{N}_{1}+\mathcal{N}_{2}\right)^{2} \\
& \mathcal{N}_{7}=\frac{\mathcal{N}_{5}}{2 \mathcal{N}_{1}}\left(\mathcal{N}_{1}+\mathcal{N}_{2}\right)+\sqrt{\mathcal{N}_{5} \mathcal{N}_{6}}
\end{aligned}
$$

Therefore, the positivity of $\mathcal{N}_{6}$ and $\mathcal{N}_{7}$ follows from other conditions, and the no-ghost condition is simply

$$
\mathcal{N}_{a}>0, \quad(a=1, \ldots, 5) .
$$

\section{F. Sound speeds}

The squared sound speeds $c_{s}^{2}$ of the five modes are determined by

$$
\begin{aligned}
0= & \lim _{\epsilon \rightarrow 0} \operatorname{det}\left[c_{s}^{2} \tilde{\mathbf{K}}-2 i \sqrt{c_{s}^{2}} \tilde{\mathbf{M}} / p-\tilde{\mathbf{V}} / p^{2}\right] \\
= & \tilde{K}_{1} \tilde{K}_{2} \tilde{K}_{3} \tilde{K}_{4} \tilde{K}_{5} \times\left[\left(c_{s}^{2}\right)^{2}-2 \alpha_{1} c_{s}^{2}+\alpha_{2}\right] \\
& \times\left[\left(c_{s}^{2}\right)^{3}-3 \alpha_{3}\left(c_{s}^{2}\right)^{2}+3 \alpha_{4} c_{s}^{2}-\alpha_{5}\right],
\end{aligned}
$$

where

$$
\begin{aligned}
\alpha_{1}= & \frac{b^{2} \mathcal{N}_{1} \mathcal{N}_{3} \mathcal{S}_{1}+2 \mathcal{N}_{2}\left(\mathcal{N}_{1}+2 \mathcal{N}_{2}\right) \mathcal{S}_{2}}{2 b^{2} \mathcal{N}_{1} \mathcal{N}_{2} \mathcal{N}_{3}} \frac{p_{x}^{2}}{p^{2}} \\
& +\frac{b^{2} \mathcal{N}_{4} \mathcal{S}_{1}+2\left(\mathcal{N}_{1}+2 \mathcal{N}_{2}\right) \mathcal{S}_{2}}{2 b^{2} \mathcal{N}_{1} \mathcal{N}_{4}} \frac{p_{\perp}^{2}}{p^{2}} \\
\alpha_{2}= & \frac{2\left[\left(\mathcal{N}_{1}+2 \mathcal{N}_{2}\right) p_{x}^{2}+3 \mathcal{N}_{2} p_{\perp}^{2}\right]\left(\mathcal{N}_{3} p_{\perp}^{2}+\mathcal{N}_{4} p_{x}^{2}\right)}{b^{2} \mathcal{N}_{1} \mathcal{N}_{2} \mathcal{N}_{3} \mathcal{N}_{4} p^{4}} \mathcal{S}_{1} \mathcal{S}_{2} \\
\alpha_{3}= & \frac{\mathcal{N}_{1} \mathcal{S}_{3} p_{x}^{2}+\mathcal{N}_{2} \mathcal{S}_{4} p_{\perp}^{2}}{3 b^{2} \mathcal{N}_{1}^{2} \mathcal{N}_{2} \mathcal{N}_{3} \mathcal{N}_{5} p^{2}} \\
\alpha_{4}= & \frac{\mathcal{S}_{5} p_{x}^{4}+2\left(\mathcal{S}_{7}-\sqrt{\mathcal{S}_{5} \mathcal{S}_{6}}\right) p_{x}^{2} p_{\perp}^{2}+\mathcal{S}_{6} p_{\perp}^{4}}{3 b^{2} \mathcal{N}_{1} \mathcal{N}_{2} \mathcal{N}_{3} \mathcal{N}_{5} p^{4}} \\
\alpha_{5}= & \frac{\mathcal{S}_{9} p_{x}^{4}+2\left(\mathcal{S}_{11}-\sqrt{\mathcal{S}_{9} \mathcal{S}_{10}}\right) p_{x}^{2} p_{\perp}^{2}+\mathcal{S}_{10} p_{\perp}^{4}}{b^{2} \mathcal{N}_{1} \mathcal{N}_{2} \mathcal{N}_{3} \mathcal{N}_{5} p^{6}} \\
& \times\left[2\left(\mathcal{N}_{1}+\mathcal{N}_{2}\right) \mathcal{S}_{2} p_{x}^{2}+b^{2} \mathcal{N}_{1} \mathcal{S}_{8} p_{\perp}^{2}\right]
\end{aligned}
$$

and $\mathcal{S}_{1, \ldots, 11}$ are given in Appendix C.

For the absence of gradient instabilities, it is necessary and sufficient to impose

$\alpha_{1}>0, \quad \alpha_{2}>0, \quad \alpha_{3}>0, \quad \alpha_{4}>0, \quad \alpha_{5}>0$,

$$
\text { for }{ }^{\forall} p_{\perp} \text { and }{ }^{\forall} p_{x} \text { such that } p^{2} \neq 0 \text {, }
$$

and 


$$
\begin{aligned}
& \alpha_{1}^{2}-\alpha_{2}>0, \quad \alpha_{3}^{2}-\alpha_{4}>0, \\
& -\alpha_{5}^{2}+2\left(3 \alpha_{4}-2 \alpha_{3}^{2}\right) \alpha_{3} \alpha_{5}+\left(3 \alpha_{3}^{2}-4 \alpha_{4}\right) \alpha_{4}^{2}>0, \\
& \text { for }{ }^{\forall} p_{\perp} \quad \text { and } \quad{ }^{\forall} p_{x} \text { such that } p^{2} \neq 0 .
\end{aligned}
$$

Here, we supposed that the no-ghost condition (3.27) is satisfied. The condition (3.30) is equivalent to

$$
\mathcal{S}_{a}>0, \quad(a=1, \ldots, 11) .
$$

\section{LONG WAVELENGTH PERTURBATIONS}

In the previous section, we studied the stability of the de Sitter solution with a homogeneous magnetic field in the UV limit, i.e., the limit where the wavelengths of perturbations are sufficiently shorter than the size of the de Sitter horizon. In this section, we study the stability of the solution in the opposite limit, namely, the limit where the wavelengths of perturbations are sufficiently longer than the size of the horizon.

As argued in Ref. [9], instabilities for modes with $p^{2}=$ $\mathcal{O}\left(H_{0}^{2}\right)$ can be as harmless as the standard Jeans instability. It is nonetheless necessary to impose the stability for those modes with $p^{2} \ll H_{0}^{2}$ if one wants the background under investigation to be realized dynamically as an attractor of the system. In general relativity, the standard Jeans instability prevents the matter-dominated FLRW background from being an attractor of the system, but this is not a problem since it is not the matter-dominated epoch but the inflationary epoch that sets the initial condition of our local patch of the Universe. On the other hand, for the system under investigation in the present paper, we would like the homogeneous magnetic field to be realized dynamically and to be sustained during a de Sitter phase representing the inflationary epoch. For this reason, we require the solution to be an attractor of the system. Namely, we require the solution to be stable against perturbations with $p^{2} \ll H_{0}^{2}$.

For this purpose, we send $p_{\perp} / H_{0}$ and $p_{x} / H_{0}$ to zero after obtaining equations of motion for properly normalized dynamical variables. Since the vector harmonics $Y_{n, p}^{\text {odd/even }}$ $(n=c c, c s, s c, s s ; p, q=2,3)$ vanish in the limit and $Y_{n, p}^{\text {odd }} /$ even $/ k_{\perp}$ remain finite, we introduce $\tilde{h}_{1, n}^{\text {odd }} \equiv k_{\perp} h_{1, n}^{\text {odd }}$, $\tilde{A}_{n}^{\text {odd }} \equiv k_{\perp} A_{n}^{\text {odd }}$, and $\tilde{V}_{n}^{\text {odd/even }} \equiv k_{\perp} V_{n}^{\text {odd/even }}$. The stability of the background solution against anisotropic perturbations with long wavelengths can then be analyzed by studying the equations of motion for the dynamical variables ( $\tilde{h}_{1, n}^{\text {odd }}, A_{1, n}, \pi_{n}, \psi_{n}, \tilde{A}_{n}^{\text {odd }}$ ) in the limit where $p_{\perp} / H_{0}$ and $p_{x} / H_{0}$ are sent to zero, after eliminating nondynamical variables. For simplicity, we set $N(t)=1$ in the rest of this section.

The equation of motion for $\tilde{h}_{1, n}^{\text {odd }}$ in the long wavelength limit does not involve other variables and is

$$
\ddot{\tilde{h}}_{1, n}^{\text {odd }}+3 H_{0} \dot{\tilde{h}}_{1, n}^{\text {odd }}=0,
$$

where an overdot represents a derivative with respect to the time variable $t$. This gives the solution

$$
\tilde{h}_{1, n}^{\text {odd }}=C_{1}+C_{2} e^{-3 H_{0} t},
$$

where $C_{1}$ and $C_{2}$ are constants. The first solution, which is constant in space and time, is actually a pure gauge. Therefore, $\tilde{h}_{1, n}^{\text {odd }}$ is stable.

The equation of motion for $A_{1, n}$ in the long wavelength limit also does not involve other variables and is

$$
\ddot{A}_{1, n}+7 H_{0} \dot{A}_{1, n}+6 H_{0}^{2} A_{1, n}=0,
$$

which gives the solution

$$
A_{1, n}=C_{3} e^{-H_{0} t}+C_{4} e^{-6 H_{0} t},
$$

where $C_{3}$ and $C_{4}$ are constants. Therefore, $A_{1, n}$ is stable.

The equations of motion for $\pi_{n}$ and $\psi_{n}$ in the long wavelength limit are coupled but can be solved easily by setting

$$
\pi_{n}=\pi_{n}^{0} e^{\Gamma H_{0} t}, \quad \psi_{n}=\psi_{n}^{0} e^{\Gamma H_{0} t},
$$

where $\pi_{n}^{0}$ and $\psi_{n}^{0}$ are constants. The general solution is of course a linear combination of solutions of this form for all allowed values of $\Gamma$. By substituting (4.5) to the equations of motion for $\pi_{n}$ and $\psi_{n}$, one obtains

$$
\Gamma(\Gamma+3)\left(\Gamma^{2}+3 \Gamma+\frac{\mathcal{A}}{\mathcal{N}}\right)=0,
$$

where $\mathcal{A}$ and $\mathcal{N}$ are the same as those defined in (2.17) and thus in particular $\mathcal{N}=\mathcal{N}_{5}$ [see (3.24)]. For $\Gamma=0, \pi_{n}^{0}=0$, and $\psi_{n}$ is a pure gauge. Therefore, $\pi_{n}$ and $\psi_{n}$ are also stable, provided that the attractor condition (2.20) and the no-ghost condition (3.27) are satisfied.

The equation of motion for $\tilde{A}_{n}^{\text {odd }}$ in the long wavelength limit does not involve other variables and is

$$
\begin{aligned}
& \ddot{\tilde{A}}_{n}^{\text {odd }}+5 H_{0} \dot{\tilde{A}}_{n}^{\text {odd }}+\mathcal{B} H_{0}^{2} \tilde{A}_{n}^{\text {odd }}=0, \\
& \mathcal{B}=\frac{\mathcal{A}_{1} k_{x}^{4}+2\left(\mathcal{A}_{3}-\sqrt{\mathcal{A}_{1} \mathcal{A}_{2}}\right) k_{x}^{2} k_{\perp}^{2}+\mathcal{A}_{2} k_{\perp}^{4}}{\left[\left(\zeta_{1}-\zeta_{3}\right) k_{x}^{2}+\left(3 g_{h} b^{2}+\zeta_{1}-\zeta_{3}\right) k_{\perp}^{2}\right]^{2}},
\end{aligned}
$$

where $\mathcal{A}_{1,2,3}$ are given in Appendix D. Therefore, $\tilde{A}_{n}^{\text {odd }}$ is stable if and only if

$$
\mathcal{A}_{a}>0, \quad(a=1,2,3) .
$$

\section{EXAMPLES}

In this section, we first show a couple of classes of models that always violate either (3.27) or (3.32). After that, we show some concrete examples of stable models 
with specific choices of parameters, which satisfy not only (3.27) and (3.32) but also (3.31), (2.20), and (4.8).

\section{A. Unstable classes of models}

Let us first consider the case without Horndeski's nonminimal vector coupling $(\xi=0)$. Setting $g_{h}=0$, we obtain $\mathcal{S}_{2}=0$. This implies that $\alpha_{2}=0$ and thus one of the five modes has vanishing sound speed.

Let us next consider the case with Horndeski's nonminimal vector coupling $(\xi \neq 0)$ but without Horndeski scalar terms. Setting $g_{3 x}=g_{3 x x}=g_{4 x}=g_{4 x x}=g_{5 x}=g_{5 x x}=0$, we obtain $\mathcal{S}_{9} / \mathcal{S}_{2}=-\left(2 \mathcal{N}_{2}-\mathcal{N}_{1}\right)^{2} \mathcal{N}_{2}$, which is nonpositive if we impose the no-ghost condition. Therefore, either $\mathcal{S}_{9}$ or $\mathcal{S}_{2}$ is nonpositive if the no-ghost condition is imposed.

These two unstable examples imply that the stability requires both Horndeski's nonminimal vector coupling and Horndeski scalar terms.

\section{B. Stable models}

Our strategy here is to find a set of parameters that satisfies (3.27) and (3.32) and then to check if (3.31), (2.20), and (4.8) are also satisfied.

We have already assumed (2.8). For simplicity, in all the examples considered in this subsection, we further set

$$
\begin{aligned}
0 & =g_{3 x x}=g_{4 x x}=g_{4 x x x}=g_{5 x} \\
& =g_{5 x x}=g_{5 x x x}=l_{y y}=l_{x w}=l_{z} .
\end{aligned}
$$

In each of the following examples, we specify $\left(g_{h}, g_{4}, g_{4 x}\right.$, $\left.g_{3 x}, b, l_{x x}, l_{w w}\right)$. We then solve the background equations of motion (2.16) with respect to $\left(l, l_{x}, l_{w}\right)$, where

$$
L=l M_{\mathrm{Pl}}^{2} H_{0}^{2}, \quad L_{X}=l_{x} M_{\mathrm{Pl}}^{2}, \quad L_{W}=l_{w} M_{\mathrm{Pl}}^{2},
$$

and it is understood that the left-hand sides of (5.2) are evaluated at the de Sitter solution with a homogeneous magnetic field. After that, we confirm that (3.27) and (3.32) as well as (3.31), (2.20), and (4.8) are satisfied.

For the set of parameters $\left(g_{h}, g_{4}, g_{4 x}, g_{3 x}, b, l_{x x}, l_{w w} ; l, l_{x}\right.$, $l_{w}$ ) and the assumptions (2.8) and (5.1), one can easily reconstruct the Lagrangian by assuming a simple ansatz and noting that $X=2 H_{0}^{2}$ and $W=-b^{2} H_{0}^{2} / 2$ on the de Sitter solution parametrized by $\left(H_{0}, b\right)$. For example, if we assume that $L$ is quadratic in $X$ and $W$ and independent of $Y$ and $Z$, then

$$
\begin{aligned}
\frac{L}{M_{\mathrm{Pl}}^{2}}= & \left(l-2 l_{x}+2 l_{x x}+\frac{1}{2} b^{2} l_{w}+\frac{1}{8} b^{4} l_{w w}\right) H_{0}^{2} \\
& +\left(l_{x}-2 l_{x x}\right) X+\left(l_{w}+\frac{1}{2} b^{2} l_{w w}\right) W \\
& +\frac{1}{2 H_{0}^{2}}\left(l_{x x} X^{2}+l_{w w} W^{2}\right) .
\end{aligned}
$$

The simplest choice of $G_{3,4,5}$ is

$$
G_{3}=g_{3 x} \frac{M_{\mathrm{Pl}}^{2}}{H_{0}^{2}} X, \quad G_{4}=g_{4} M_{\mathrm{Pl}}^{2}+g_{4 x} \frac{M_{\mathrm{Pl}}^{2}}{H_{0}^{2}} X, \quad G_{5}=0 .
$$

The value of $\xi$ is

$$
\xi=g_{h} \frac{H_{0}^{2}}{M_{\mathrm{Pl}}^{2}} .
$$

\section{Example 1}

Let us consider the following choice of parameters,

$$
\begin{aligned}
g_{h} & =-1, \quad g_{4}=\frac{1}{2}, \quad g_{4 x}=0, \quad g_{3 x}=\frac{1}{10}, \\
b & =\frac{1}{10}, \quad l_{x x}=-\frac{1}{10}, \quad l_{w w}=-1,
\end{aligned}
$$

for which the background equations of motion (2.16) give

$$
l=-\frac{79}{25}, \quad l_{x}=-\frac{31}{50}, \quad l_{w}=20 .
$$

In this case, we have

$$
\begin{array}{ll}
\mathcal{N}_{1}=\frac{12}{25}, & \mathcal{N}_{2}=\frac{1}{2}, \quad \mathcal{N}_{3}=12, \\
\mathcal{N}_{4}=12, & \mathcal{N}_{5}=\frac{1408}{15625},
\end{array}
$$

and

$$
\begin{aligned}
& \mathcal{S}_{1}=\frac{1}{2}, \quad \mathcal{S}_{2}=\frac{1}{50}, \quad \mathcal{S}_{3}=\frac{59456}{9765625}, \\
& \mathcal{S}_{4}=\frac{35469972}{6103515625}, \quad \mathcal{S}_{5}=\frac{1696}{390625}, \quad \mathcal{S}_{6}=\frac{7940769}{1953125000}, \\
& \mathcal{S}_{7}=\frac{2060321}{488281250}+\sqrt{\mathcal{S}_{5} \mathcal{S}_{6}}, \quad \mathcal{S}_{8}=\frac{1201}{100}, \quad \mathcal{S}_{9}=\frac{224}{15625}, \\
& \mathcal{S}_{10}=\frac{29}{2500}, \quad \mathcal{S}_{11}=\frac{823}{62500}+\sqrt{\mathcal{S}_{9} \mathcal{S}_{10}} .
\end{aligned}
$$

Thus, Eqs. (3.27) and (3.32) are satisfied.

The conditions (2.20) and (4.8) are also satisfied as

$$
\begin{aligned}
& \mathcal{A}=\frac{5979}{390625}, \quad \mathcal{A}_{1}=\frac{256}{625}, \quad \mathcal{A}_{2}=\frac{87451}{250000}, \\
& \mathcal{A}_{3}=\frac{47493}{125000}+\sqrt{\mathcal{A}_{1} \mathcal{A}_{2}} .
\end{aligned}
$$

After substituting (5.9) into (3.29) to obtain explicit expressions of $\alpha_{1, \ldots, 5}$, it is straightforward to compute the three expressions on the left-hand sides of the inequalities in (3.31) and to show that they all have the form $A / B$, 
where $A$ and $B$ are polynomials of $p_{\perp}^{2}$ and $p_{x}^{2}$ with positive coefficients. Therefore, Eq. (3.31) is also satisfied.

\section{Example 2}

Let us consider the following choice of parameters.

$$
\begin{aligned}
g_{h}=-1, & g_{4}=\frac{1}{2}, \quad g_{4 x}=0, \quad g_{3 x}=\frac{1}{10}, \\
b=\frac{1}{10}, & l_{x x}=-\frac{1}{10}, \quad l_{w w}=0,
\end{aligned}
$$

for which the background equations of motion (2.16) give the same values of $l, l_{x}$, and $l_{w}$ as shown in (5.7).

In this case, we have

$$
\begin{array}{ll}
\mathcal{N}_{1}=\frac{12}{25}, & \mathcal{N}_{2}=\frac{1}{2}, \quad \mathcal{N}_{3}=12 \\
\mathcal{N}_{4}=12, & \mathcal{N}_{5}=\frac{1408}{15625},
\end{array}
$$

and

$$
\begin{aligned}
& \mathcal{S}_{1}=\frac{1}{2}, \quad \mathcal{S}_{2}=\frac{1}{50}, \quad \mathcal{S}_{3}=\frac{59456}{9765625}, \\
& \mathcal{S}_{4}=\frac{1418292}{244140625}, \quad \mathcal{S}_{5}=\frac{1696}{390625}, \quad \mathcal{S}_{6}=\frac{79353}{19531250}, \\
& \mathcal{S}_{7}=\frac{16477}{3906250}+\sqrt{\mathcal{S}_{5} \mathcal{S}_{6}}, \quad \mathcal{S}_{8}=12, \quad \mathcal{S}_{9}=\frac{224}{15625} \\
& \mathcal{S}_{10}=\frac{29}{2500}, \quad \mathcal{S}_{11}=\frac{823}{62500}+\sqrt{\mathcal{S}_{9} \mathcal{S}_{10}} .
\end{aligned}
$$

Thus, Eqs. (3.27) and (3.32) are satisfied.

The conditions (2.20) and (4.8) are also satisfied as

$$
\begin{aligned}
\mathcal{A} & =\frac{239}{15625}, \quad \mathcal{A}_{1}=\frac{256}{625}, \quad \mathcal{A}_{2}=\frac{1749}{5000}, \\
\mathcal{A}_{3} & =\frac{5699}{15000}+\sqrt{\mathcal{A}_{1} \mathcal{A}_{2}} .
\end{aligned}
$$

After substituting (5.18) into (3.29) to obtain explicit expressions of $\alpha_{1, \ldots, 5}$, it is straightforward to compute the three expressions on the left-hand sides of the inequalities in (3.31) and to show that they all have the form $A / B$, where $A$ and $B$ are polynomials of $p_{\perp}^{2}$ and $p_{x}^{2}$ with positive coefficients. Therefore, Eq. (3.31) is also satisfied.

\section{Example 3}

Let us consider the following choice of parameters,

$$
\begin{aligned}
& g_{h}=-\frac{1}{20}, \quad g_{4}=\frac{1}{2} \quad g_{4 x}=0, \quad g_{3 x}=\frac{1}{10}, \\
& b=\frac{1}{10}, \quad l_{x x}=-\frac{1}{10}, \quad l_{w w}=0,
\end{aligned}
$$

for which the background equations of motion (2.16) give

$$
l=-\frac{376}{125}, \quad l_{x}=-\frac{601}{1000}, \quad l_{w}=1 .
$$

In this case, we have

$$
\begin{aligned}
& \mathcal{N}_{1}=\frac{499}{1000}, \quad \mathcal{N}_{2}=\frac{1}{2}, \quad \mathcal{N}_{3}=\frac{3}{5}, \\
& \mathcal{N}_{4}=\frac{3}{5}, \quad \mathcal{N}_{5}=\frac{108990799}{1000000000},
\end{aligned}
$$

and

$$
\begin{aligned}
& \mathcal{S}_{1}=\frac{1}{2}, \quad \mathcal{S}_{2}=\frac{1}{1000}, \quad \mathcal{S}_{3}=\frac{371370384707}{1000000000000000} \\
& \mathcal{S}_{4}=\frac{92615485451247}{250000000000000000}, \quad \mathcal{S}_{5}=\frac{50621001581}{200000000000000} \\
& \mathcal{S}_{6}=\frac{252272242903}{1000000000000000}, \\
& \mathcal{S}_{7}=\frac{50538124821}{200000000000000}+\sqrt{\mathcal{S}_{5} \mathcal{S}_{6}}, \quad \mathcal{S}_{8}=\frac{3}{5} \\
& \mathcal{S}_{9}=\frac{29949199}{2000000000}, \quad \mathcal{S}_{10}=\frac{59301}{4000000} \\
& \mathcal{S}_{11}=\frac{59601699}{4000000000}+\sqrt{\mathcal{S}_{9} \mathcal{S}_{10}} .
\end{aligned}
$$

Thus, Eqs. (3.27) and (3.32) are satisfied.

The conditions (2.20) and (4.8) are also satisfied as

$$
\begin{aligned}
\mathcal{A} & =\frac{304101}{500000000}, \quad \mathcal{A}_{1}=\frac{90601}{250000}, \quad \mathcal{A}_{2}=\frac{718809}{2000000}, \\
\mathcal{A}_{3} & =\frac{90045653}{249500000}+\sqrt{\mathcal{A}_{1} \mathcal{A}_{2}} .
\end{aligned}
$$

After substituting (5.13) into (3.29) to obtain explicit expressions of $\alpha_{1, \ldots, 5}$, it is straightforward to compute the three expressions on the left-hand sides of the inequalities in (3.31) and to show that they all have the form $A / B$, where $A$ and $B$ are polynomials of $p_{\perp}^{2}$ and $p_{x}^{2}$ with positive coefficients. Therefore, Eq. (3.31) is also satisfied.

\section{Example 4}

Let us consider the following choice of parameters,

$$
\begin{aligned}
& g_{h}=-\frac{1}{20}, \quad g_{4}=\frac{1}{2}, \quad g_{4 x}=-\frac{1}{10}, \quad g_{3 x}=0, \\
& b=\frac{1}{10}, \quad l_{x x}=-\frac{1}{10}, \quad l_{w w}=0,
\end{aligned}
$$

for which the background equations of motion (2.16) give 


$$
l=-\frac{676}{125}, \quad l_{x}=\frac{599}{1000}, \quad l_{w}=1 .
$$

In this case, we have

$$
\begin{aligned}
& \mathcal{N}_{1}=\frac{899}{1000}, \quad \mathcal{N}_{2}=\frac{9}{10}, \quad \mathcal{N}_{3}=\frac{3}{5}, \\
& \mathcal{N}_{4}=\frac{3}{5}, \quad \mathcal{N}_{5}=\frac{108704599}{1000000000},
\end{aligned}
$$

and

$$
\begin{aligned}
& \mathcal{S}_{1}=\frac{1}{2}, \quad \mathcal{S}_{2}=\frac{1}{1000}, \quad \mathcal{S}_{3}=\frac{9143271446151}{5000000000000000}, \\
& \mathcal{S}_{4}=\frac{456009833453647}{250000000000000000}, \quad \mathcal{S}_{5}=\frac{9305798288929}{5000000000000000} \\
& \mathcal{S}_{6}=\frac{9284212649127}{5000000000000000}, \\
& \mathcal{S}_{7}=\frac{9295017384133}{5000000000000000}+\sqrt{\mathcal{S}_{5} \mathcal{S}_{6}}, \quad \mathcal{S}_{8}=\frac{3}{5} \\
& \mathcal{S}_{9}=\frac{207508199}{2000000000}, \quad \mathcal{S}_{10}=\frac{10351541}{100000000} \\
& \mathcal{S}_{11}=\frac{414541899}{4000000000}+\sqrt{\mathcal{S}_{9} \mathcal{S}_{10}}
\end{aligned}
$$

Thus, Eqs. (3.27) and (3.32) are satisfied.

The conditions (2.20) and (4.8) are also satisfied as

$$
\begin{aligned}
\mathcal{A} & =\frac{9185101}{500000000}, \quad \mathcal{A}_{1}=\frac{1692601}{250000}, \quad \mathcal{A}_{2}=\frac{13530009}{2000000}, \\
\mathcal{A}_{3} & =\frac{3042086553}{449500000}+\sqrt{\mathcal{A}_{1} \mathcal{A}_{2}} .
\end{aligned}
$$

After substituting (5.23) into (3.29) to obtain explicit expressions of $\alpha_{1, \ldots, 5}$, it is straightforward to compute the three expressions on the left-hand sides of the inequalities in (3.31) and to show that they all have the form $A / B$, where $A$ and $B$ are polynomials of $p_{\perp}^{2}$ and $p_{x}^{2}$ with positive coefficients. Therefore, Eq. (3.31) is also satisfied.

\section{SUMMARY AND DISCUSSION}

We have presented a detailed stability analysis for the de Sitter solution with a homogeneous magnetic field that was recently found in Ref. [5] in the context of a $U(1)$ gauge theory nonminimally coupled to scalar-tensor gravity. The magnetic field is stealth in the sense that the corresponding stress-energy tensor is of the form of an effective cosmological constant and thus is isotropic despite the fact that the magnetic field has a preferred spatial direction. We have studied the stability of the solution against linear perturbations in the subhorizon and superhorizon limits and have shown some explicit examples that satisfy all stability conditions. Stable models include both Horndeski's nonminimal vector coupling and the Horndeski scalar terms.
The stable de Sitter solution with a homogeneous magnetic field opens up a new possibility for inflationary magnetogenesis, in which magnetic fields in the Universe at all scales may originate from a classical, homogeneous magnetic field sustained during inflation. Toward such a new scenario of inflationary magnetogenesis, an important step forward is to show a graceful exit from the de Sitter solution with constant values of $(X, W, Y, Z, \chi)$. In particular, the scalar field $\phi$ should be stabilized around a local minimum of its potential with a sufficiently large mass at late time after inflation in order to recover the standard Einstein-Maxwell theory. (To be more precise, what is recovered is the Einstein-Maxwell theory with the Horndeski's nonminimal vector coupling.) Also, as already pointed out in Ref. [5], a field other than $\phi$ needs to be introduced as the main source of curvature perturbation, i.e., either an inflaton or a curvaton, in order to avoid too large statistical anisotropies and non-Gaussianities. One eventually needs to establish the stability of the whole system all the way from the inflationary epoch to the standard expansion history at late time through the graceful exit. While the stability in the superhorizon limit is not necessarily required after inflation (see the second paragraph of Sec. IV), the stability in the subhorizon limit is required not only during but also after inflation. The present paper has established the stability of the inflationary epoch and thus can be considered as the first step toward the new inflationary magnetogenesis scenario based on a classical, homogeneous magnetic field sustained during inflation.

The recent multimessenger detection of binary neutron stars put a strong constraint on the deviation of the propagation speed of gravitational waves from that of light at the present $[10,11]$. If the Horndeski scalar-tensor theory is used as a model of the late-time acceleration of the Universe then the functions $G_{4}$ and $G_{5}$ are strongly constrained. On the other hand, the propagation speed of gravitational waves in the early Universe such as the inflationary epoch is not constrained at all by such observations. Therefore, the model considered in Ref. [5] and in the present paper is consistent with the multimessenger detection of binary neutron stars as far as the scalar field is stabilized around a local minimum of its potential with a sufficiently large mass at late time after inflation, as required anyway for the recovery of the Einstein-Maxwell theory (with Horndeski's nonminimal vector coupling).

After inflation and the stabilization of $\phi$ around a local minimum of its potential, the homogeneous magnetic field is no longer stealth, and thus the background spacetime is then described by a Bianchi I geometry (instead of a FLRW one). It is therefore important to investigate phenomenology of the Bianchi I universe with a homogeneous magnetic field and the standard content of the universe (radiation, matter, and the cosmological constant) in the context of the Einstein-Maxwell theory with Horndeski's nonminimal vector coupling [12]. 


\section{ACKNOWLEDGMENTS}

The author thanks Axel Brandenburg, Ruth Durrer, Yiwen Huang, Tina Kahniashvili, and Sayan Mandal for helpful discussions. A part of the work was completed during the author's visits to Simon Fraser University, University of Alberta, and University of Victoria. The author is therefore grateful to Andrei Frolov, Valeri Frolov, Werner Israel, Maxim Pospelov, and Andrei Zelnikov for their warm hospitality. The work was supported in part by Japan Society for the Promotion of Science (JSPS) Grants-in-Aid for Scientific Research (KAKENHI) Grants No. 17H02890 and No. 17H06359 and by World Premier International Research Center Initiative (WPI), MEXT, Japan.

\section{APPENDIX A: EXPLICIT EXPRESSIONS OF SUBMATRICES}

The submatrices in (3.21) are

$$
\begin{aligned}
& \mathbf{K}_{\mathrm{odd}}=\left(\begin{array}{cc}
\zeta_{6} & 0 \\
0 & \zeta_{1}
\end{array}\right) \\
& \mathbf{K}_{\mathrm{even}}=\left(\begin{array}{ccc}
\zeta_{7} & 0 & 0 \\
0 & \zeta_{2} & \zeta_{3} \\
0 & \zeta_{3} & 0
\end{array}\right) \text {, } \\
& \mathbf{M}_{\text {even }}=H_{0}\left(\begin{array}{ccc}
0 & 0 & 0 \\
0 & 0 & -b^{2} g_{h} \\
0 & b^{2} g_{h} & 0
\end{array}\right) \text {, } \\
& \mathbf{M}_{\text {mix }}=p_{\perp}\left(\begin{array}{ccc}
0 & w_{1} & 2 b g_{h} \\
-2 b g_{h} & 0 & 0
\end{array}\right), \\
& \mathbf{V}_{\text {odd }}=p_{\perp}^{2}\left(\begin{array}{cc}
\zeta_{5} & 0 \\
0 & g_{4}
\end{array}\right)+p_{x}^{2}\left(\begin{array}{cc}
-12 g_{h} & 0 \\
0 & 0
\end{array}\right) \\
& +H_{0}^{2}\left(\begin{array}{cc}
-4 \zeta_{6} & 0 \\
0 & 0
\end{array}\right) \text {, } \\
& \mathbf{V}_{\text {even }}=p_{\perp}^{2}\left(\begin{array}{ccc}
-12 g_{h} & 0 & 0 \\
0 & w_{2} & w_{3} \\
0 & w_{3} & 0
\end{array}\right)+p_{x}^{2}\left(\begin{array}{ccc}
0 & 0 & 0 \\
0 & w_{4} & 0 \\
0 & 0 & 0
\end{array}\right) \\
& +H_{0}^{2}\left(\begin{array}{ccc}
-4 \zeta_{7} & 0 & 0 \\
0 & w_{5} & 3 b^{2} g_{h} \\
0 & 3 b^{2} g_{h} & 0
\end{array}\right) \text {, } \\
& \mathbf{V}_{\text {mix }}=p_{\perp} H_{0}\left(\begin{array}{ccc}
0 & w_{6} & 2 b g_{h} \\
8 b g_{h} & 0 & 0
\end{array}\right) \text {, } \\
& \mathbf{A}_{\text {odd }}=p_{x} H_{0}\left(\begin{array}{ll}
0 & -4 b^{2} g_{h}
\end{array}\right),
\end{aligned}
$$

$$
\begin{aligned}
& \mathbf{A}_{\text {even }}=\frac{p_{\perp}^{3}}{H_{0}}\left(\begin{array}{ccc}
0 & 0 & 0 \\
0 & 0 & 0 \\
0 & 0 & 0 \\
0 & 8 b^{2} g_{h}-2 \zeta_{3} & \zeta_{1}
\end{array}\right)+\frac{p_{\perp} p_{x}^{2}}{H_{0}}\left(\begin{array}{ccc}
0 & 0 & 0 \\
0 & 0 & 0 \\
0 & 0 & 0 \\
0 & -2 \zeta_{3} & 0
\end{array}\right) \\
& +p_{\perp} H_{0}\left(\begin{array}{ccc}
0 & b^{2}\left(2 g_{h}+\frac{1}{2} \zeta_{6}\right) & -2 b^{2} g_{h} \\
0 & 0 & 0 \\
0 & 0 & 0 \\
0 & -b \zeta_{4} & 0
\end{array}\right) \\
& +p_{x} H_{0}\left(\begin{array}{ccc}
0 & 0 & 0 \\
-\zeta_{7} & 0 & 0 \\
0 & -4 b^{2} g_{h} & 0 \\
0 & 0 & 0
\end{array}\right) \\
& \mathbf{A}_{\text {mix I }}=p_{\perp} p_{x}\left(\begin{array}{lll}
4 b g_{h} & 0 & 0
\end{array}\right), \\
& \mathbf{A}_{\text {mix II }}=p_{\perp}^{2}\left(\begin{array}{cc}
0 & 0 \\
0 & 0 \\
0 & 0 \\
\zeta_{4} & 0
\end{array}\right)+p_{\perp} p_{x}\left(\begin{array}{cc}
0 & 0 \\
0 & 0 \\
4 b g_{h} & 0 \\
0 & 0
\end{array}\right) \\
& +p_{x}^{2}\left(\begin{array}{cc}
4 b g_{h} & 0 \\
0 & 0 \\
0 & 0 \\
0 & 0
\end{array}\right)+H_{0}^{2}\left(\begin{array}{cc}
b \zeta_{6} & 0 \\
0 & 0 \\
0 & 0 \\
0 & 0
\end{array}\right) \\
& \mathbf{B}_{\text {odd }}=p_{x}\left(\begin{array}{ll}
0 & -\zeta_{1}
\end{array}\right), \\
& \mathbf{B}_{\mathrm{even}}=p_{\perp}\left(\begin{array}{ccc}
0 & -8 b^{2} g_{h}+2 \zeta_{3} & -\zeta_{1} \\
0 & 0 & 0 \\
0 & 0 & 0 \\
0 & w_{7} & 2\left(\zeta_{1}-\zeta_{3}\right)
\end{array}\right) \\
& +p_{x}\left(\begin{array}{ccc}
0 & 0 & 0 \\
\zeta_{7} & 0 & 0 \\
0 & -2 \zeta_{3} & 0 \\
0 & 0 & 0
\end{array}\right) \text {, } \\
& \mathbf{B}_{\text {mix }}=H_{0}\left(\begin{array}{cc}
-b \zeta_{6} & 0 \\
0 & 0 \\
0 & 0 \\
0 & 0
\end{array}\right) \text {, } \\
& \mathbf{C}_{\text {odd }}=p_{\perp}^{2}\left(-2 b^{2} g_{h}+\zeta_{1}\right)+p_{x}^{2}\left(\zeta_{1}\right)+H_{0}^{2}\left(b^{2} \zeta_{6}\right),
\end{aligned}
$$




$$
\begin{aligned}
\mathbf{C}_{\text {even }}= & p_{\perp}^{2}\left(\begin{array}{cccc}
0 & 0 & 0 & w_{8} \\
0 & \zeta_{6} & 0 & 0 \\
0 & 0 & \zeta_{1} & 0 \\
w_{8} & 0 & 0 & w_{9}
\end{array}\right) \\
& +p_{\perp} p_{x}\left(\begin{array}{cccc}
0 & 0 & \zeta_{1} & 0 \\
0 & 0 & 0 & 0 \\
\zeta_{1} & 0 & 0 & 4\left(\zeta_{3}-\zeta_{1}\right) \\
0 & 0 & 4\left(\zeta_{3}-\zeta_{1}\right) & 0
\end{array}\right) \\
& +p_{x}^{2}\left(\begin{array}{cccc}
\zeta_{1} & 0 & 0 & 0 \\
0 & \zeta_{7} & 0 & 0 \\
0 & 0 & 0 & 0 \\
0 & 0 & 0 & 0
\end{array}\right)+H_{0}^{2}\left(\begin{array}{cccc}
b^{2} \zeta_{6} & 0 & 0 & 0 \\
0 & 0 & 0 & 0 \\
0 & 0 & 0 & 0 \\
0 & 0 & 0 & 0
\end{array}\right)
\end{aligned}
$$

(A16)

$$
\mathbf{C}_{\text {mix }}=p_{\perp} H_{0}\left(\begin{array}{llll}
0 & b \zeta_{6} & 0 & 0
\end{array}\right),
$$

where

$$
\begin{aligned}
& w_{1}=-b\left(2 g_{h}+\frac{1}{4} \zeta_{6}\right)+\frac{1}{4} \zeta_{4}, \\
& w_{2}=-b^{2}\left(7 g_{h}+\frac{1}{4} \zeta_{6}\right)+\zeta_{1}+\zeta_{3}-g_{4}, \\
& w_{3}=b^{2} g_{h}-\frac{1}{2} \zeta_{1}+\frac{1}{2} g_{4}, \\
& w_{4}=-4 b^{2} g_{h}+\zeta_{1}+\zeta_{3}-g_{4}, \\
& w_{5}=\left(-6 g_{h}+\zeta_{5}\right) b^{2}+\frac{3}{2} b \zeta_{4}, \\
& w_{6}=-b\left(2 g_{h}+\zeta_{5}+\zeta_{6}\right)-\frac{1}{2} \zeta_{4}, \\
& w_{7}=16 b^{2} g_{h}-2\left(\zeta_{2}+3 \zeta_{3}\right), \\
& w_{8}=12 b^{2} g_{h}+4\left(\zeta_{1}-\zeta_{3}\right), \\
& w_{9}=-56 b^{2} g_{h}+4\left(-3 \zeta_{1}+\zeta_{2}+6 \zeta_{3}\right) .
\end{aligned}
$$

\section{APPENDIX B: COEFFICIENTS OF MATRIX COMPONENTS}

The coefficients $c_{1}, \ldots, c_{17}$ in (3.23) are as follows:

$$
\begin{aligned}
c_{1}= & \zeta_{1}\left(\zeta_{1} \zeta_{2}+3 \zeta_{3}^{2}\right)-2 b^{2} g_{h}\left(\zeta_{1} \zeta_{2}+8 \zeta_{1} \zeta_{3}+2 \zeta_{3}^{2}\right) \\
& +b^{4} g_{h}^{2}\left(16 \zeta_{1}+\zeta_{2}+24 \zeta_{3}\right)-32 b^{6} g_{h}^{3}, \\
c_{2}= & \zeta_{1}\left(\zeta_{1} \zeta_{2}+3 \zeta_{3}^{2}\right)-b^{2} g_{h}\left(\zeta_{1} \zeta_{2}+16 \zeta_{1} \zeta_{3}+\zeta_{3}^{2}\right) \\
& +2 b^{4} g_{h}^{2} \zeta_{3}\left(8-\frac{\zeta_{3}}{\zeta_{1}}\right) \\
c_{3}= & \frac{1}{4} \zeta_{1}\left(\zeta_{4}-b \zeta_{6}\right)+\frac{1}{4} b g_{h}\left[-8\left(\zeta_{1}-\zeta_{3}\right)-b \zeta_{4}+b^{2} \zeta_{6}\right] \\
& -6 b^{3} g_{h}^{2},
\end{aligned}
$$

$$
c_{4}=\frac{1}{4} \zeta_{1}\left(\zeta_{4}-b \zeta_{6}\right)-2 b g_{h}\left(\zeta_{1}-\zeta_{3}\right)-4 b^{3} g_{h}^{2}\left(4-\frac{\zeta_{3}}{\zeta_{1}}\right)
$$

$$
\begin{aligned}
c_{5}= & \zeta_{1}\left[\left(\zeta_{1} \zeta_{2}+3 \zeta_{3}^{2}\right)-\frac{b}{8}\left(\zeta_{4}-b \zeta_{6}\right)\left(4 \zeta_{1}-\zeta_{3}\right)\right] \\
& +b^{2} g_{h} \zeta_{1}\left(4 \zeta_{1}-\zeta_{2}-21 \zeta_{3}\right)+32 b^{4} g_{h}^{2} \zeta_{1},
\end{aligned}
$$$$
c_{6}=\zeta_{1}\left(\zeta_{1} \zeta_{2}+3 \zeta_{3}^{2}\right)-2 b^{2} g_{h} \zeta_{3}\left(8 \zeta_{1}-\zeta_{3}\right)
$$

$c_{7}=-\frac{1}{4} \zeta_{1}^{2} \zeta_{6} b^{2}-\left(\zeta_{1}-\zeta_{3}\right)^{2} g_{4}+\left(\zeta_{1}-\zeta_{3}\right) \zeta_{1}^{2}$

$$
\begin{aligned}
& +\left[\frac{b^{2}}{2} \zeta_{1} \zeta_{6}+6\left(\zeta_{3}-\zeta_{1}\right) g_{4}-\zeta_{1}\left(\zeta_{1}-4 \zeta_{3}\right)\right] b^{2} g_{h} \\
& -\left[\frac{1}{4} \zeta_{6} b^{2}+3\left(3 \zeta_{1}+3 g_{4}+\zeta_{3}\right)\right] b^{4} g_{h}^{2}+9 b^{6} g_{h}^{3},
\end{aligned}
$$

$$
\begin{aligned}
c_{8}= & -\frac{b^{2}}{2} \zeta_{1}^{2} \zeta_{6}-3\left(\zeta_{1}-\zeta_{3}\right)^{2} g_{4}+3\left(\zeta_{1}-\zeta_{3}\right) \zeta_{1}^{2} \\
& +\left[\frac{b^{2}}{2} \zeta_{1} \zeta_{6}+12\left(\zeta_{3}-\zeta_{1}\right) g_{4}+6 \zeta_{1}\left(2 \zeta_{3}-\zeta_{1}\right)\right] b^{2} g_{h} \\
& -\left(17 \zeta_{1}+7 \zeta_{3}+9 g_{4}\right) b^{4} g_{h}^{2}+12 b^{6} g_{h}^{3},
\end{aligned}
$$

$$
\begin{aligned}
c_{9}= & -\frac{b^{2}}{4} \zeta_{1}^{2} \zeta_{6}-3\left(\zeta_{1}-\zeta_{3}\right)^{2} g_{4}+3\left(\zeta_{1}-\zeta_{3}\right) \zeta_{1}^{2} \\
& +3\left[2\left(\zeta_{3}-\zeta_{1}\right) g_{4}+\zeta_{1}\left(4 \zeta_{3}-3 \zeta_{1}\right)\right] b^{2} g_{h} \\
& -4\left(2 \zeta_{1}+\zeta_{3}\right) b^{4} g_{h}^{2},
\end{aligned}
$$




$$
\begin{aligned}
& c_{11}=\frac{1}{4} \zeta_{1}^{2} g_{4}\left(\zeta_{1} \zeta_{2}+3 \zeta_{3}^{2}\right)^{2}-\zeta_{1}\left(\zeta_{1} \zeta_{2}+3 \zeta_{3}^{2}\right)\left(\zeta_{1} \zeta_{2}+8 \zeta_{1} \zeta_{3}+2 \zeta_{3}^{2}\right) g_{4} b^{2} g_{h} \\
& +\left[-\frac{1}{16} \zeta_{1}^{2} \zeta_{6}\left(4 \zeta_{1}-\zeta_{3}\right)^{2} b^{2}+\left(-4 \zeta_{1}^{4}+10 \zeta_{1}^{3} \zeta_{2}+10 \zeta_{1}^{3} \zeta_{3}+\frac{5}{4} \zeta_{1}^{2} \zeta_{2}^{2}+\frac{51}{2} \zeta_{1}^{2} \zeta_{3} \zeta_{2}+\frac{343}{4} \zeta_{1}^{2} \zeta_{3}^{2}\right.\right. \\
& \left.\left.+\frac{9}{2} \zeta_{1} \zeta_{3}^{2} \zeta_{2}+63 \zeta_{1} \zeta_{3}^{3}+3 \zeta_{3}^{4}\right) g_{4}+\frac{1}{4} \zeta_{1}^{2}\left(4 \zeta_{1}-\zeta_{3}\right)\left(4 \zeta_{1}^{2}-2 \zeta_{1} \zeta_{2}-5 \zeta_{1} \zeta_{3}-5 \zeta_{3}^{2}\right)\right] b^{4} g_{h}^{2} \\
& +\left[\frac{1}{4} \zeta_{1} \zeta_{6}\left(4 \zeta_{1}-\zeta_{3}\right)^{2} b^{2}+\left(-16 \zeta_{1}^{3}-30 \zeta_{1}^{2} \zeta_{2}-144 \zeta_{1}^{2} \zeta_{3}-\frac{41}{2} \zeta_{1} \zeta_{3} \zeta_{2}-219 \zeta_{1} \zeta_{3}^{2}-\frac{1}{2} \zeta_{1} \zeta_{2}^{2}-\zeta_{3}^{2} \zeta_{2}-30 \zeta_{3}^{3}\right) g_{4}\right. \\
& \left.-\frac{1}{4} \zeta_{1}\left(4 \zeta_{1}-\zeta_{3}\right)\left(12 \zeta_{1}^{2}-10 \zeta_{1} \zeta_{2}-59 \zeta_{1} \zeta_{3}-20 \zeta_{3}^{2}\right)\right] b^{6} g_{h}^{3} \\
& +\left[-\frac{1}{4} \zeta_{6}\left(4 \zeta_{1}-\zeta_{3}\right)^{2} b^{2}+\left(112 \zeta_{1}^{2}+20 \zeta_{1} \zeta_{2}+296 \zeta_{1} \zeta_{3}+3 \zeta_{3} \zeta_{2}+95 \zeta_{3}^{2}\right) g_{4}-\left(4 \zeta_{1}-\zeta_{3}\right)\left(16 \zeta_{1}^{2}+4 \zeta_{1} \zeta_{2}+44 \zeta_{1} \zeta_{3}+5 \zeta_{3}^{2}\right)\right] b^{8} g_{h}^{4} \\
& +\left[-32\left(4 \zeta_{1}+3 \zeta_{3}\right) g_{4}+\left(4 \zeta_{1}-\zeta_{3}\right)\left(68 \zeta_{1}+2 \zeta_{2}+39 \zeta_{3}\right)\right] b^{10} g_{h}^{5}+64\left(\zeta_{3}-4 \zeta_{1}\right) b^{12} g_{h}^{6}, \\
& c_{12}=\frac{3}{4} \zeta_{1}^{2} g_{4}\left(\zeta_{1} \zeta_{2}+3 \zeta_{3}^{2}\right)^{2}-\zeta_{1}\left(\zeta_{1} \zeta_{2}+3 \zeta_{3}^{2}\right)\left(2 \zeta_{1} \zeta_{2}+24 \zeta_{1} \zeta_{3}+3 \zeta_{3}^{2}\right) g_{4} b^{2} g_{h} \\
& +\left[\left(-4 \zeta_{1}^{4}+10 \zeta_{1}^{3} \zeta_{2}+10 \zeta_{1}^{3} \zeta_{3}+\frac{5}{4} \zeta_{1}^{2} \zeta_{2}^{2}+\frac{1}{2} \zeta_{1} \zeta_{3}^{2} \zeta_{2}+\frac{115}{2} \zeta_{1}^{2} \zeta_{3} \zeta_{2}+\frac{855}{4} \zeta_{1}^{2} \zeta_{3}^{2}+127 \zeta_{1} \zeta_{3}^{3}-7 \zeta_{3}^{4}\right) g_{4}\right. \\
& \left.+\frac{1}{4} \zeta_{1}^{2}\left(4 \zeta_{1}-\zeta_{3}\right)\left(4 \zeta_{1}^{2}-2 \zeta_{1} \zeta_{2}-5 \zeta_{1} \zeta_{3}-5 \zeta_{3}^{2}\right)\right] b^{4} g_{h}^{2} \\
& +\left[\left(16 \zeta_{1}^{3}-20 \zeta_{1}^{2} \zeta_{2}-200 \zeta_{1}^{2} \zeta_{3}-27 \zeta_{1} \zeta_{3} \zeta_{2}-415 \zeta_{1} \zeta_{3}^{2}+3 \zeta_{3}^{2} \zeta_{2}+8 \zeta_{3}^{3}+4 \frac{\zeta_{3}^{4}}{\zeta_{1}}\right) g_{4}\right. \\
& \left.-\zeta_{1}\left(4 \zeta_{1}-\zeta_{3}\right)\left(8 \zeta_{1}^{2}-2 \zeta_{1} \zeta_{2}-18 \zeta_{1} \zeta_{3}-3 \zeta_{3}^{2}\right)\right] b^{6} g_{h}^{3} \\
& +\left[\left(-16 \zeta_{1}^{2}+360 \zeta_{1} \zeta_{3}+39 \zeta_{3}^{2}-12 \frac{\zeta_{3}^{3}}{\zeta_{1}}\right) g_{4}+\left(4 \zeta_{1}-\zeta_{3}\right)\left(20 \zeta_{1}^{2}-2 \zeta_{1} \zeta_{2}-57 \zeta_{1} \zeta_{3}+3 \zeta_{3}^{2}\right)\right] b^{8} g_{h}^{4} \\
& -4\left(4-\frac{\zeta_{3}}{\zeta_{1}}\right)\left(4 \zeta_{1}^{2}-13 \zeta_{1} \zeta_{3}+2 \zeta_{3}^{2}\right) b^{10} g_{h}^{5},
\end{aligned}
$$

$c_{13}=\frac{3}{4} \zeta_{1}^{2} g_{4}\left(\zeta_{1} \zeta_{2}+3 \zeta_{3}^{2}\right)^{2}-\zeta_{1}^{2}\left(\zeta_{1} \zeta_{2}+3 \zeta_{3}^{2}\right)\left(\zeta_{2}+24 \zeta_{3}\right) g_{4} b^{2} g_{h}+\zeta_{3}\left(8 \zeta_{1}-\zeta_{3}\right)\left(4 \zeta_{1} \zeta_{2}+24 \zeta_{1} \zeta_{3}+9 \zeta_{3}^{2}\right) g_{4} b^{4} g_{h}^{2}$ $-4 \frac{\zeta_{3}^{2}}{\zeta_{1}}\left(8 \zeta_{1}-\zeta_{3}\right)^{2} g_{4} b^{6} g_{h}^{3}$

$$
c_{14}=\frac{1}{4} \zeta_{1}^{2} g_{4}\left(\zeta_{1} \zeta_{2}+3 \zeta_{3}^{2}\right)^{2}-\zeta_{1} \zeta_{3}\left(\zeta_{1} \zeta_{2}+3 \zeta_{3}^{2}\right)\left(8 \zeta_{1}-\zeta_{3}\right) g_{4} b^{2} g_{h}+\zeta_{3}^{2}\left(8 \zeta_{1}-\zeta_{3}\right)^{2} g_{4} b^{4} g_{h}^{2}
$$

$$
\begin{aligned}
c_{15}= & \frac{1}{8} \zeta_{1}^{2} \zeta_{6}\left(4 \zeta_{1}-\zeta_{3}\right) b^{2}+\frac{1}{2}\left(4 \zeta_{1}^{2}-\zeta_{1} \zeta_{2}-5 \zeta_{1} \zeta_{3}-2 \zeta_{3}^{2}\right)\left[\left(\zeta_{1}-\zeta_{3}\right) g_{4}-\zeta_{1}^{2}\right] \\
& +\left[-\frac{3}{8} \zeta_{1} \zeta_{6}\left(4 \zeta_{1}-\zeta_{3}\right) b^{2}+\left(10 \zeta_{1}^{2}-\zeta_{1} \zeta_{2}-\frac{5}{2} \zeta_{1} \zeta_{3}-\frac{1}{2} \zeta_{3} \zeta_{2}-12 \zeta_{3}^{2}\right) g_{4}+\zeta_{1}\left(4 \zeta_{1}^{2}-2 \zeta_{1} \zeta_{2}-19 \zeta_{1} \zeta_{3}-\frac{5}{2} \zeta_{3}^{2}\right)\right] b^{2} g_{h} \\
& +\left[\frac{1}{4} \zeta_{6}\left(4 \zeta_{1}-\zeta_{3}\right) b^{2}+\left(-4 \zeta_{1}+\frac{3}{2} \zeta_{2}+43 \zeta_{3}\right) g_{4}+26 \zeta_{1}^{2}+\frac{5}{2} \zeta_{1} \zeta_{2}+\frac{71}{2} \zeta_{1} \zeta_{3}+\zeta_{3}^{2}\right] b^{4} g_{h}^{2} \\
& +\left(-68 \zeta_{1}-48 g_{4}-\zeta_{2}-15 \zeta_{3}\right) b^{6} g_{h}^{3}+32 b^{8} g_{h}^{4},
\end{aligned}
$$




$$
\begin{aligned}
c_{16}= & \frac{1}{16} \zeta_{1}^{2} \zeta_{6}\left(4 \zeta_{1}-\zeta_{3}\right) b^{2}+\frac{1}{2}\left(4 \zeta_{1}^{2}-\zeta_{1} \zeta_{2}-5 \zeta_{1} \zeta_{3}-2 \zeta_{3}^{2}\right)\left(\zeta_{1}-\zeta_{3}\right) g_{4}-\frac{1}{2}\left(4 \zeta_{1}^{2}-\zeta_{1} \zeta_{2}-5 \zeta_{1} \zeta_{3}-2 \zeta_{3}^{2}\right) \zeta_{1}^{2} \\
& +\left[-\frac{1}{8} \zeta_{1} \zeta_{6}\left(4 \zeta_{1}-\zeta_{3}\right) b^{2}+\frac{1}{4}\left(12 \zeta_{1}^{2}-2 \zeta_{1} \zeta_{2}+29 \zeta_{1} \zeta_{3}-\zeta_{3} \zeta_{2}-54 \zeta_{3}^{2}+4 \frac{\zeta_{3}^{3}}{\zeta_{1}}\right) g_{4}+\frac{1}{4} \zeta_{1}\left(32 \zeta_{1}^{2}-6 \zeta_{1} \zeta_{2}-84 \zeta_{1} \zeta_{3}-3 \zeta_{3}^{2}\right)\right] b^{2} g_{h} \\
& +\left[-\frac{1}{2 \zeta_{1}}\left(28 \zeta_{1}^{2}-55 \zeta_{1} \zeta_{3}+6 \zeta_{3}^{2}\right) g_{4}+4 \zeta_{1}^{2}+\zeta_{1} \zeta_{2}+37 \zeta_{1} \zeta_{3}-\frac{7}{2} \zeta_{3}^{2}\right] b^{4} g_{h}^{2}-2\left(12 \zeta_{1}+5 \zeta_{3}-\frac{\zeta_{3}^{2}}{\zeta_{1}}\right) b^{6} g_{h}^{3} \\
c_{17}= & \frac{1}{2}\left(4 \zeta_{1}^{2}-\zeta_{1} \zeta_{2}-5 \zeta_{1} \zeta_{3}-2 \zeta_{3}^{2}\right)\left(\zeta_{1}-\zeta_{3}\right) g_{4}-\frac{1}{2}\left(4 \zeta_{1}^{2}-\zeta_{1} \zeta_{2}-5 \zeta_{1} \zeta_{3}-2 \zeta_{3}^{2}\right) \zeta_{1}^{2} \\
& +\left[-\left(1-\frac{\zeta_{3}}{\zeta_{1}}\right)\left(4 \zeta_{1}^{2}-13 \zeta_{1} \zeta_{3}+2 \zeta_{3}^{2}\right) g_{4}+\zeta_{1}\left(12 \zeta_{1}^{2}-\zeta_{1} \zeta_{2}-23 \zeta_{1} \zeta_{3}+\zeta_{3}^{2}\right)\right] b^{2} g_{h}+2\left(-8 \zeta_{1}^{2}+18 \zeta_{1} \zeta_{3}-3 \zeta_{3}^{2}\right) b^{4} g_{h}^{2}
\end{aligned}
$$

\section{APPENDIX C: COEFFICIENTS OF DISPERSION RELATION}

In this Appendix, we show the coefficients $\mathcal{S}_{1, \ldots, 11}$ of the dispersion relation (3.28), (3.29):

$$
\begin{gathered}
\mathcal{S}_{1}=g_{4}, \\
\mathcal{S}_{2}=\mathcal{N}_{2}-\mathcal{N}_{1},
\end{gathered}
$$

$$
\begin{aligned}
\mathcal{S}_{3}= & -b^{2} g_{4} \mathcal{N}_{1} \mathcal{N}_{2} \mathcal{N}_{3} \zeta_{3}^{2}-\mathcal{N}_{1} \mathcal{N}_{3}\left(\mathcal{N}_{1}^{2} \mathcal{N}_{2}-\mathcal{N}_{5}\right) b^{2} g_{4}+\left[2 \mathcal{N}_{3} \mathcal{N}_{2} \mathcal{N}_{1}^{2} b^{2} g_{4}+\mathcal{N}_{3} \mathcal{N}_{2} \mathcal{N}_{1}^{2}\left(\mathcal{N}_{1}-2 \mathcal{N}_{2}\right) b^{2}\right] \zeta_{3} \\
& -\mathcal{N}_{1}^{3} \mathcal{N}_{2} \mathcal{N}_{3}\left(\mathcal{N}_{1}-2 \mathcal{N}_{2}\right) b^{2}-2 \mathcal{N}_{2} \mathcal{N}_{5}\left(\mathcal{N}_{1}+2 \mathcal{N}_{2}\right)\left(\mathcal{N}_{1}-\mathcal{N}_{2}\right),
\end{aligned}
$$

$$
\begin{aligned}
& \mathcal{S}_{4}=[\left.-\mathcal{N}_{1} \mathcal{N}_{3}\left(2 \mathcal{N}_{1}-\mathcal{N}_{2}\right) b^{2} g_{4}+4\left(2 \mathcal{N}_{1}-\mathcal{N}_{2}\right)^{2}\left(\mathcal{N}_{1}-\mathcal{N}_{2}\right)^{2}\right] \zeta_{3}^{2} \\
&+ {\left[2 \mathcal{N}_{1}^{2}\left(2 \mathcal{N}_{1}-\mathcal{N}_{2}\right)\left(\mathcal{N}_{1}-\mathcal{N}_{2}\right) b \zeta_{4}+\mathcal{N}_{3} \mathcal{N}_{1}^{2}\left(7 \mathcal{N}_{1}-5 \mathcal{N}_{2}\right) b^{2} g_{4}-\mathcal{N}_{3} \mathcal{N}_{1}^{2}\left(3 \mathcal{N}_{1}^{2}-3 \mathcal{N}_{1} \mathcal{N}_{2}+\mathcal{N}_{2}^{2}\right) b^{2}\right.} \\
&-\left.8 \mathcal{N}_{1}\left(2 \mathcal{N}_{1}-\mathcal{N}_{2}\right)\left(5 \mathcal{N}_{1}-4 \mathcal{N}_{2}\right)\left(\mathcal{N}_{1}-\mathcal{N}_{2}\right)^{2}\right] \zeta_{3}+b^{2} \mathcal{N}_{1}^{4} \zeta_{4}^{2} / 4 \\
&+\left[-b^{3} \mathcal{N}_{1}^{4} \mathcal{N}_{3} / 2-2 \mathcal{N}_{1}^{3}\left(5 \mathcal{N}_{1}-4 \mathcal{N}_{2}\right)\left(\mathcal{N}_{1}-\mathcal{N}_{2}\right) b\right] \zeta_{4}+b^{2} \mathcal{N}_{1}^{2} \mathcal{N}_{5} \zeta_{5}-\mathcal{N}_{1} \mathcal{N}_{3}\left(5 \mathcal{N}_{1}^{3}-4 \mathcal{N}_{1}^{2} \mathcal{N}_{2}-\mathcal{N}_{5}\right) b^{2} g_{4} \\
&+\mathcal{N}_{1}^{3} \mathcal{N}_{3}\left(15 \mathcal{N}_{1}^{2}-21 \mathcal{N}_{1} \mathcal{N}_{2}+8 \mathcal{N}_{2}^{2}\right) b^{2} / 2+4\left(\mathcal{N}_{1}-\mathcal{N}_{2}\right)^{2}\left(25 \mathcal{N}_{1}^{4}-40 \mathcal{N}_{1}^{3} \mathcal{N}_{2}+16 \mathcal{N}_{1}^{2} \mathcal{N}_{2}^{2}+\mathcal{N}_{2} \mathcal{N}_{5}\right) \\
& \mathcal{S}_{5}=\left[-b^{2} \mathcal{N}_{1} \mathcal{N}_{3} g_{4}^{2}+2 \mathcal{N}_{2}\left(\mathcal{N}_{1}+2 \mathcal{N}_{2}\right)\left(\mathcal{N}_{1}-\mathcal{N}_{2}\right) g_{4}\right] \zeta_{3}^{2} \\
&+\left\{2 \mathcal{N}_{3} \mathcal{N}_{1}^{2} b^{2} g_{4}^{2}+\left[\mathcal{N}_{3} \mathcal{N}_{1}^{2}\left(\mathcal{N}_{1}-2 \mathcal{N}_{2}\right) b^{2}-4 \mathcal{N}_{1} \mathcal{N}_{2}\left(\mathcal{N}_{1}+2 \mathcal{N}_{2}\right)\left(\mathcal{N}_{1}-\mathcal{N}_{2}\right)\right] g_{4}\right. \\
&\left.-2 \mathcal{N}_{1} \mathcal{N}_{2}\left(\mathcal{N}_{1}+2 \mathcal{N}_{2}\right)\left(\mathcal{N}_{1}-\mathcal{N}_{2}\right)\left(\mathcal{N}_{1}-2 \mathcal{N}_{2}\right)\right\} \zeta_{3}-b^{2} g_{4}^{2} \mathcal{N}_{1}^{3} \mathcal{N}_{3} \\
&+\left[-\mathcal{N}_{1}^{3} \mathcal{N}_{3}\left(\mathcal{N}_{1}-2 \mathcal{N}_{2}\right) b^{2}+2\left(\mathcal{N}_{1}+2 \mathcal{N}_{2}\right)\left(\mathcal{N}_{1}-\mathcal{N}_{2}\right)\left(\mathcal{N}_{1}^{2} \mathcal{N}_{2}-\mathcal{N}_{5}\right)\right] g_{4} \\
&+2 \mathcal{N}_{1}^{2} \mathcal{N}_{2}\left(\mathcal{N}_{1}+2 \mathcal{N}_{2}\right)\left(\mathcal{N}_{1}-\mathcal{N}_{2}\right)\left(\mathcal{N}_{1}-2 \mathcal{N}_{2}\right)
\end{aligned}
$$

$$
\begin{aligned}
\mathcal{S}_{6}= & {\left[-\mathcal{N}_{2}\left(2 \mathcal{N}_{1}-\mathcal{N}_{2}\right) b^{2} g_{4} \zeta_{5}-b^{2} \mathcal{N}_{1} \mathcal{N}_{3} g_{4}^{2}\right] \zeta_{3}^{2} } \\
& +\left\{2 \mathcal{N}_{1} \mathcal{N}_{2}\left(\mathcal{N}_{1}-\mathcal{N}_{2}\right) b g_{4} \zeta_{4}+\left[\mathcal{N}_{1} \mathcal{N}_{2}\left(7 \mathcal{N}_{1}-5 \mathcal{N}_{2}\right) b^{2} g_{4}+\mathcal{N}_{1} \mathcal{N}_{2}\left(\mathcal{N}_{1}^{2}-3 \mathcal{N}_{1} \mathcal{N}_{2}+\mathcal{N}_{2}^{2}\right) b^{2}\right] \zeta_{5}\right. \\
& \left.+\mathcal{N}_{1} \mathcal{N}_{3}\left(5 \mathcal{N}_{1}-3 \mathcal{N}_{2}\right) b^{2} g_{4}^{2}-\mathcal{N}_{1} \mathcal{N}_{2} \mathcal{N}_{3}\left(2 \mathcal{N}_{1}-\mathcal{N}_{2}\right) b^{2} g_{4}-4 \mathcal{N}_{1} \mathcal{N}_{2}^{2}\left(\mathcal{N}_{1}-\mathcal{N}_{2}\right)^{2}\right\} \zeta_{3}+b^{2} g_{4} \mathcal{N}_{1}^{2} \mathcal{N}_{2} \zeta_{4}^{2} / 4 \\
& +\left\{\left[-b^{3} \mathcal{N}_{1}^{2} \mathcal{N}_{2} \mathcal{N}_{3} / 2-\mathcal{N}_{1} \mathcal{N}_{2}\left(5 \mathcal{N}_{1}-3 \mathcal{N}_{2}\right)\left(\mathcal{N}_{1}-\mathcal{N}_{2}\right) b\right] g_{4}-\mathcal{N}_{1} \mathcal{N}_{2}^{2}\left(\mathcal{N}_{1}-\mathcal{N}_{2}\right)^{2} b\right\} \zeta_{4} \\
& +\left[-\mathcal{N}_{2}\left(5 \mathcal{N}_{1}^{3}-4 \mathcal{N}_{1}^{2} \mathcal{N}_{2}-\mathcal{N}_{5}\right) b^{2} g_{4}-b^{4} \mathcal{N}_{1}^{3} \mathcal{N}_{2} \mathcal{N}_{3} / 4-\mathcal{N}_{1}^{2} \mathcal{N}_{2}\left(5 \mathcal{N}_{1}^{2}-15 \mathcal{N}_{1} \mathcal{N}_{2}+8 \mathcal{N}_{2}^{2}\right) b^{2} / 2\right] \zeta_{5} \\
& -\mathcal{N}_{1} \mathcal{N}_{3}\left(5 \mathcal{N}_{1}-3 \mathcal{N}_{2}\right)^{2} b^{2} g_{4}^{2} / 4+\mathcal{N}_{1} \mathcal{N}_{2} \mathcal{N}_{3}\left(2 \mathcal{N}_{1}-\mathcal{N}_{2}\right)\left(5 \mathcal{N}_{1}-3 \mathcal{N}_{2}\right) b^{2} g_{4} / 2-\mathcal{N}_{1} \mathcal{N}_{2}^{2} \mathcal{N}_{3}\left(\mathcal{N}_{1}-\mathcal{N}_{2}\right)^{2} b^{2} / 4 \\
& +2 \mathcal{N}_{1} \mathcal{N}_{2}^{2}\left(5 \mathcal{N}_{1}-3 \mathcal{N}_{2}\right)\left(\mathcal{N}_{1}-\mathcal{N}_{2}\right)^{2}
\end{aligned}
$$




$$
\begin{aligned}
& \mathcal{S}_{7}=\left[-b^{2} g_{4} \mathcal{N}_{1} \mathcal{N}_{2} \zeta_{5} / 2-b^{2} \mathcal{N}_{1} \mathcal{N}_{3} g_{4}^{2}+\left(\mathcal{N}_{1}-\mathcal{N}_{2}\right)\left(-\mathcal{N}_{2}+4 \mathcal{N}_{1}\right)\left(2 \mathcal{N}_{1}^{2}-3 \mathcal{N}_{1} \mathcal{N}_{2}+2 \mathcal{N}_{2}^{2}\right) g_{4} / \mathcal{N}_{1}\right] \zeta_{3}^{2} \\
& +\left\{\mathcal{N}_{1}\left(2 \mathcal{N}_{1}-\mathcal{N}_{2}\right)\left(\mathcal{N}_{1}-\mathcal{N}_{2}\right) b g_{4} \zeta_{4}+\left[b^{2} g_{4} \mathcal{N}_{1}^{2} \mathcal{N}_{2}+\mathcal{N}_{1}^{2} \mathcal{N}_{2}\left(\mathcal{N}_{1}-2 \mathcal{N}_{2}\right) b^{2} / 2\right] \zeta_{5}+\mathcal{N}_{3} \mathcal{N}_{1}\left(7 \mathcal{N}_{1}-3 \mathcal{N}_{2}\right) b^{2} g_{4}^{2} / 2\right. \\
& +\left[-\mathcal{N}_{3} \mathcal{N}_{1}\left(3 \mathcal{N}_{1}^{2}-4 \mathcal{N}_{1} \mathcal{N}_{2}+3 \mathcal{N}_{2}^{2}\right) b^{2} / 2-\left(\mathcal{N}_{1}-\mathcal{N}_{2}\right)\left(40 \mathcal{N}_{1}^{3}-85 \mathcal{N}_{1}^{2} \mathcal{N}_{2}+73 \mathcal{N}_{1} \mathcal{N}_{2}^{2}-22 \mathcal{N}_{2}^{3}\right)\right] g_{4} \\
& \left.-\mathcal{N}_{2}\left(\mathcal{N}_{1}-\mathcal{N}_{2}\right)\left(\mathcal{N}_{1}^{3}-3 \mathcal{N}_{1}^{2} \mathcal{N}_{2}+\mathcal{N}_{1} \mathcal{N}_{2}^{2}-2 \mathcal{N}_{2}^{3}\right)\right\} \zeta_{3}+b^{2} g_{4} \mathcal{N}_{1}^{3} \zeta_{4}^{2} / 8 \\
& +\left[-\mathcal{N}_{1}^{3} \mathcal{N}_{3} b^{3} / 4-\mathcal{N}_{1}^{2}\left(5 \mathcal{N}_{1}-4 \mathcal{N}_{2}\right)\left(\mathcal{N}_{1}-\mathcal{N}_{2}\right) b\right] g_{4} \zeta_{4}+\left[-\mathcal{N}_{1}\left(\mathcal{N}_{1}^{2} \mathcal{N}_{2}-\mathcal{N}_{5}\right) b^{2} g_{4}-\mathcal{N}_{1}^{3} \mathcal{N}_{2}\left(\mathcal{N}_{1}-2 \mathcal{N}_{2}\right) b^{2}\right] \zeta_{5} / 2 \\
& -\mathcal{N}_{1}^{2} \mathcal{N}_{3}\left(5 \mathcal{N}_{1}-3 \mathcal{N}_{2}\right) b^{2} g_{4}^{2} / 2+\left[\mathcal{N}_{1}^{2} \mathcal{N}_{3}\left(15 \mathcal{N}_{1}^{2}-23 \mathcal{N}_{1} \mathcal{N}_{2}+12 \mathcal{N}_{2}^{2}\right) b^{2} / 4+\left(\mathcal{N}_{1}-\mathcal{N}_{2}\right)\left(50 \mathcal{N}_{1}^{5}-125 \mathcal{N}_{1}^{4} \mathcal{N}_{2}\right.\right. \\
& \left.\left.+116 \mathcal{N}_{1}^{3} \mathcal{N}_{2}^{2}-38 \mathcal{N}_{1}^{2} \mathcal{N}_{2}^{3}-\mathcal{N}_{1} \mathcal{N}_{2} \mathcal{N}_{5}-2 \mathcal{N}_{2}^{2} \mathcal{N}_{5}\right) / \mathcal{N}_{1}\right] g_{4}+\mathcal{N}_{1}^{2} \mathcal{N}_{2} \mathcal{N}_{3}\left(\mathcal{N}_{1}+2 \mathcal{N}_{2}\right)\left(\mathcal{N}_{1}-\mathcal{N}_{2}\right) b^{2} / 4 \\
& +\mathcal{N}_{1} \mathcal{N}_{2}\left(\mathcal{N}_{1}-\mathcal{N}_{2}\right)\left(5 \mathcal{N}_{1}^{3}-9 \mathcal{N}_{1}^{2} \mathcal{N}_{2}-10 \mathcal{N}_{1} \mathcal{N}_{2}^{2}+8 \mathcal{N}_{2}^{3}\right) / 2+\sqrt{\mathcal{S}_{5} \mathcal{S}_{6}}, \\
& \mathcal{S}_{8}=\zeta_{5}, \\
& \mathcal{S}_{9}=-g_{4}\left(\mathcal{N}_{1}-\zeta_{3}\right)\left(-\zeta_{3} g_{4}+g_{4} \mathcal{N}_{1}+\mathcal{N}_{1}^{2}-2 \mathcal{N}_{1} \mathcal{N}_{2}\right) \\
& \mathcal{S}_{10}=-\zeta_{3}^{2} g_{4}^{2}+\left[\left(5 \mathcal{N}_{1}-3 \mathcal{N}_{2}\right) g_{4}^{2}-g_{4} \mathcal{N}_{2}^{2}\right] \zeta_{3}-\left(5 \mathcal{N}_{1}-3 \mathcal{N}_{2}\right)^{2} g_{4}^{2} / 4+\left[-b^{2} \mathcal{N}_{1} \mathcal{N}_{2} \mathcal{N}_{3} / 4+\mathcal{N}_{2}^{2}\left(5 \mathcal{N}_{1}-3 \mathcal{N}_{2}\right) / 2\right] g_{4} \\
& -\mathcal{N}_{2}^{2}\left(\mathcal{N}_{1}-\mathcal{N}_{2}\right)^{2} / 4 \\
& \mathcal{S}_{11}=\left\{-\zeta_{3}^{2} g_{4}+\left[\left(7 \mathcal{N}_{1}-3 \mathcal{N}_{2}\right) g_{4} / 2+\mathcal{N}_{1}^{2} / 2-\mathcal{N}_{1} \mathcal{N}_{2}-\mathcal{N}_{2}^{2} / 2\right] \zeta_{3}-\mathcal{N}_{1}\left(5 \mathcal{N}_{1}-3 \mathcal{N}_{2}\right) g_{4} / 2-\mathcal{N}_{3} \mathcal{N}_{1}^{2} b^{2} / 8\right. \\
& \left.-\mathcal{N}_{1}\left(5 \mathcal{N}_{1}^{2}-13 \mathcal{N}_{1} \mathcal{N}_{2}+4 \mathcal{N}_{2}^{2}\right) / 4\right\} g_{4}+\sqrt{\mathcal{S}_{9} \mathcal{S}_{10}} \text {. }
\end{aligned}
$$

\section{APPENDIX D: COEFFICIENTS OF THE EQUATION OF MOTION IN THE LONG WAVELENGTH LIMIT}

The coefficients $\mathcal{A}_{1,2,3}$ in (4.7) are as follows:

$$
\begin{gathered}
\mathcal{A}_{1}=4\left(-\zeta_{3}+\zeta_{1}\right)^{2}, \\
\mathcal{A}_{2}=\left(36 g_{h}+7 \zeta_{5} / 2\right) g_{h} b^{4}+9 b^{3} \zeta_{4} g_{h} / 4+\left[24\left(\zeta_{1}-\zeta_{3}\right) g_{h}+\left(3 \zeta_{1}-6 \zeta_{3}-\zeta_{2}\right) \zeta_{5} / 4-\zeta_{4}^{2} / 16\right] b^{2} \\
+3 \zeta_{4}\left(-\zeta_{3}+\zeta_{1}\right) b / 4+4\left(-\zeta_{3}+\zeta_{1}\right)^{2}, \\
\mathcal{A}_{3}=28 g_{h}^{3} b^{6} / \zeta_{1}+\left(3 \zeta_{1}-2 \zeta_{2}-30 \zeta_{3}\right) g_{h}^{2} b^{4} / \zeta_{1}-g_{h} \zeta_{4}\left(-\zeta_{3}+\zeta_{1}\right) b^{3} / \zeta_{1} \\
+ \\
+\left[\left(27 \zeta_{1}^{2}+\left(3 \zeta_{2}-30 \zeta_{3}\right) \zeta_{1}+12 \zeta_{3}^{2}\right) g_{h} /\left(2 \zeta_{1}\right)+\left(-\zeta_{3}+\zeta_{1}\right)^{2} \zeta_{5} /\left(2 \zeta_{1}\right)\right] b^{2} \\
+3 \zeta_{4}\left(-\zeta_{3}+\zeta_{1}\right) b / 8+4\left(-\zeta_{3}+\zeta_{1}\right)^{2}+\sqrt{\mathcal{A}_{1} \mathcal{A}_{2}}
\end{gathered}
$$

[1] L. M. Widrow, Origin of galactic and extragalactic magnetic fields, Rev. Mod. Phys. 74, 775 (2002).

[2] A. Kandus, K. E. Kunze, and C. G. Tsagas, Primordial magnetogenesis, Phys. Rep. 505, 1 (2011).

[3] R. Durrer and A. Neronov, Cosmological magnetic fields: Their generation, evolution and observation, Astron. Astrophys. Rev. 21, 62 (2013).

[4] K. Subramanian, The origin, evolution and signatures of primordial magnetic fields, Rep. Prog. Phys. 79, 076901 (2016).
[5] S. Mukohyama, Stealth magnetic field in de Sitter spacetime, Phys. Rev. D 94, 121302 (2016).

[6] G. W. Horndeski, Second-order scalar-tensor field equations in a four-dimensional space, Int. J. Theor. Phys. 10, 363 (1974).

[7] C. Deffayet, X. Gao, D. A. Steer, and G. Zahariade, From k-essence to generalised Galileons, Phys. Rev. D 84, 064039 (2011).

[8] G. W. Horndeski, Conservation of Charge and the EinsteinMaxwell field equations, J. Math. Phys. (N.Y.) 17, 1980 (1976). 
[9] A. E. Gumrukcuoglu, S. Mukohyama, and T. P. Sotiriou, Low energy ghosts and the Jeans' instability, Phys. Rev. D 94, 064001 (2016).

[10] B. P. Abbott et al. (LIGO Scientific and Virgo Collaborations), GW170817: Observation of Gravitational Waves from a Binary Neutron Star Inspiral, Phys. Rev. Lett. 119, 161101 (2017).
[11] B. P. Abbott et al. (LIGO Scientific, Virgo, Fermi-GBM, and INTEGRAL Collaborations), Gravitational waves and gamma-rays from a binary neutron star merger: GW170817 and GRB 170817A, Astrophys. J. 848, L13 (2017).

[12] R. Durrer, Y. Huang, T. Kahniashvili, S. Mandal, and S. Mukohyama (to be published). 